\title{
SEGMENTACIÓN EDUCATIVA: UNA ESTIMACION PARA LA ÚLTIMA DÉCADA EN ARGENTINA
}

\author{
María María Ibañez Martín* \\ enviado: febrero 2015 - aceptado: mayo 2015
}

\begin{abstract}
Resumen
El abordaje de la equidad en los sistemas educativos es amplio y diverso dentro de las ciencias sociales y, en especial, en Economía de la Educación. Los orígenes de sistemas educativos inequitativos son variados, por tal motivo el objetivo del trabajo es contrastar la hipótesis de que existe desigual distribución del alumnado en función de sus condiciones socioeconómicas. Para ello, se utilizan datos del estudio la Encuesta Permanente de Hogares en el período 2004-2014 y se emplean diversas variantes de modelos Ordered Logit y Generalized Ordered Logit, de acuerdo a los resultados del test de Brant. Los resultados muestran evidencia en favor de la hipótesis propuesta.
\end{abstract}

Palabras claves: Segmentación educativa, Movilidad Social, Ordered Logit Código JEL: I24, I25

\begin{abstract}
Educational inequality is a topic widely discussed in the literature, with different factors that influence the unequal distribution of students. In that order, the study aims to test the hypothesis that there is educational segmentation around socioeconomic conditions of students. To do this, the study used data from the Permanent Household Survey for the period 2004-2013 and used model of Generalized Ordered Logit and Ordered Logit. The results show evidence for the proposed hypothesis.
\end{abstract}

Key words: Educational Segmentation, Social Mobility, Ordered Logit JEL Code: I24, I25

* IIESS-CONICET-UNS - maria.ibanez@uns.edu.ar 


\section{INTRODUCCION}

El concepto de equidad en el ámbito de la educación cuenta con diversas definiciones, en función de la dimensión que sea considerada como primordial a equiparar (Sen, 1979; Ibañez Martín, Delbianco y London, 2014).

Dentro de las concepciones de equidad, en el presente trabajo se tomará la propuesta por Sen (1979), en la que una sociedad será equitativa si las personas verifican igualdad en sus oportunidades. Las últimas involucran el acceso a los bienes, la capacidad de decisión, la habilidad de transformar el acceso en un estilo de vida digno y valorar dicha posición. Al igual que las demás teorías de equidad social basadas en igualdad de oportunidades, el punto de partida es reconocer la existencia de diferencias - sociales, económicas, culturales, sexuales, étnicas, entre otras- que afectan la capacidad de los individuos para hacer uso de los recursos en pos de mejorar sus estilos de vida (Formichella, 2010; Ibañez Martín, 2014).

A su vez, la definición de equidad en el ámbito educativo conlleva una discusión filosófica y enfrenta una importante diversidad de posturas. Tomando las capacidades como base de información, pueden encontrarse diversos criterios para definir la equidad presente en un sistema de educación: igualdad en el acceso, en los medios, en el acervo de conocimientos y en logros educativos (Marchesi et al, 2014). En este trabajo se tomará en consideración el que contempla la igualdad respecto a los logros educativos, que tiene como objetivo la impartición de una formación equivalente a todos (Blanco, 2006; Cervini 2009; Formichella, 2010); en palabras de López (2005), todas las personas deben aprender lo mismo, o que nadie pueda acceder a más conocimiento en caso que lo pretenda.

Una evaluación de la equidad podría realizarse a partir del análisis de la calidad educativa que recibe el alumnado, pudiendo diferir en función de la posición socioeconómica del alumnado, el centro al que asiste, la conformación familiar, la localización del centro, entre otros (Aranda, 2013). En caso de que las condiciones mencionadas fueran determinantes de una desigualdad en la calidad educativa, y por tanto de los logros educativos de los estudiantes, podría sostenerse que el sistema educativo se encuentra segmentado (Cervini, 2009). Por otra parte, siguiendo las ideas de Calero (1999) la equidad del sistema educativo podría estudiarse a partir de la incidencia que el sistema educativo ha tenido sobre quienes han transitado por él; en este caso, el autor reconoce la existencia de una dimensión externa de la equidad. 
El estudio empírico analiza si las condiciones mencionadas en el párrafo anterior son determinantes del destino educativo de los niños argentinos en edad de asistir a centros educativos. En otras palabras, se busca determinar si las condiciones socioeconómicas del alumnado explican la segregación público-privada, entendiendo que las calidades educativas difieren entre ambos circuitos. Con tal propósito, se realizan estimaciones de modelos logísticos ordenados, en su versión generalizada y no generalizada.

El trabajo se estructura de la siguiente manera: en la sección I, se presenta la discusión de los conceptos de equidad social y educativa. En la sección II se presentan las especificaciones de la base de datos utilizada y las estimaciones de los modelos logísticos ordenados. En la sección IV se exponen las conclusiones finales del estudio realizado.

\section{EQUIDAD EN LA EDUCACIÓN}

El concepto de equidad cuenta con diversas definiciones, en función de la dimensión que sea considerada como primordial a equiparar (Sen, 1979; Ibañez Martín, Delbianco y London, 2014). En el presente trabajo, siguiendo la postura tomada a lo largo de diversos estudios (Formichella e Ibañez Martín, 2014; Ibañez Martin y Formichella, 2014; Ibañez Martín, Delbianco y London, 2014), se definirá a la equidad a partir de la igualdad de oportunidades. Dicho criterio de equidad cuenta con diversas vertientes (Formichella, 2010) esbozadas a partir de las ideas de Roemer (1955), Arneson (1989), Cohen (1989) y Sen (1979). La diferencia entre los autores reside principalmente en la respuesta que brindan a ¿Igualdad de oportunidades respecto a qué?: Roemer (1955) hace referencia a que la equidad se verifica si las condiciones externas no están relacionadas con la existencia de resultados disímiles; Arneson (1989) referencia a "igualdad de oportunidades para el bienestar", sosteniendo que las personas deben enfrentar en la vida adulta conjuntos de elección exactamente iguales y teniendo en cuenta una cuestión potencial respecto al bienestar; y, por su parte, Cohen (1989) sintetiza sus ideas en la expresión "igualdad en el acceso a las ventajas", postulando que las personas deben verificar igualdad en el acceso a cierto resultado pero también en las habilidades necesarias para tomar decisiones sobre las oportunidades.

Por su parte, Sen (1979) postula que una sociedad será equitativa si las personas enfrentan igualdad en la posibilidad de elegir sus estilos de vida. Es decir, están en igualdad de oportunidades respecto al acceso a los bienes, la capacidad de decisión, la habilidad de transformar el acceso en un estilo de vida 
digno y valorar dicha posición. En su planteamiento, la primera noción importante es la de "funciones", definidas como las diferentes cosas que un individuo puede valorar ser o hacer. Luego, se agrega como segundo nivel a las "capabilities" o "capacidades" que pueden ser definidas como oportunidades reales y actuales que las personas tienen para tomar decisiones informadas, para poder garantizarse una vida y las actividades que tienen razones para valorar (Aristizabal et al., 2010, p.125), y son la "unidad de medida" de su concepción de equidad. Entonces, la sociedad será equitativa cuando las personas están igualmente capacitados para elegir entre diferentes estilos de vida y la libertad de hacerlo (Bolívar 2011; Formichella, 2010; Loewe, 2009).

La educación es considerada por todas las variantes de la equidad basada en igualdad de oportunidades. En particular, Sen (1979) la contempla dentro de las libertadas fundamentales enmarcándola dentro de las libertades sociales de los individuos. La falta de educación es la privación de un aspecto fundamental en la vida de un individuo; por lo que la sociedad no será equitativa si no existe igualdad en este sentido.

Por tal motivo y por la importancia que reviste dentro de su lógica de pensamiento, en el presente trabajo se tomará la propuesta de Sen (1979). Al igual que las demás teorías de equidad social basadas en igualdad de oportunidades, el punto de partida es reconocer la existencia de diferencias -sociales, económicas, culturales, sexuales, étnicas, entre otras - que afectan la capacidad de los individuos para hacer uso de los recursos en pos de mejorar sus estilos de vida (Formichella, 2011).

Como cualquier definición de un concepto amplio, la aplicación del mismo al ámbito educativo no escapa de la diversidad de posturas y la discusión. Partiendo de la adopción de la noción de Sen, y por tanto tomando las capacidades como base de información, puede tomarse como necesario de igualar: el acceso, los medios de aprendizaje, el acervo de conocimientos o los logros educativos (Marchesi et al., 2014). De ellos, el considerado será el que contempla la igualdad que verifican los alumnos respecto a los logros educativos, garantizando tanto el acceso a la educación como la permanencia en la escuela y la obtención de resultados significativos (Latapí 1993; Rivero, 1999; Muñoz Izquierdo, 2009; García Huidobro, 2005; Blanco, 2006; Formichella, 2010; 2012).

La definición de equidad educativa como la igualdad en los logros es consistente con la definición de equidad social en términos de igualdad de oportunidades, y contempla la diferencia de condiciones de origen que no es abordada por el resto de las posturas enumeradas (Ibañez Martín, 2014). Tal 
como se mencionó el criterio implica la impartición de una formación equivalente para todo el alumnado, implicando la insuficiencia respecto a la igualdad en las condiciones de acceso para que una sociedad verifique equidad educativa. También es necesario igualar las condiciones de escolarización al interior del sistema para que la trayectoria individual de los alumnos no se vea reproducida en las trayectorias educativas (Duru-Bellat y Van-Zanten y, 2001; Silva-Laya, 2012). Enguita (2013) postula que la educación cumple un rol relevante a la hora de equiparar las condiciones de la vida adulta y, por ello, la equidad en el ámbito educativo es una cuestión primordial. En este orden de ideas, López (2006) plantea que las desigualdades de origen deben ser reemplazadas por desigualdades que permitan llegar a la equidad educativa y social.

Por otra parte, la equidad educativa puede desagregarse en dos dimensiones: interna y externa (Calero, 1999). El primer concepto, equidad educativa interna, está relacionado a lo que sucede dentro del sistema educativo y con la calidad de contenidos que reciben los niños en función de la institución a la que asisten, su posición socioeconómica y su entorno familiar. La equidad educativa externa se relaciona estrechamente con el concepto de movilidad social, la posición que verifican los individuos una vez que culminan su formación, su capacidad de desarrollarse en la vida cotidiana, de acceder al mercado laboral y permanecer en él (Calero 1999; Morresi, Donnini y Cerioni, 2009; Formichella, 2010).

Una de las expresiones de la inexistencia de equidad educativa en la esfera interna es la segmentación educativa.

En sus estudios, los institutos DiNIECE y OCDE presentan evidencia respecto a que la calidad educativa a la que acceden los alumnos argentinos difiere ampliamente, comprobado a partir de los resultados de las pruebas estandarizadas (DiNIECE, 2010; OCDE, 2010; Viscardi, 2014). También Tosoni y Nantel (2010) postulan que la segmentación educativa se ha profundizado en las últimas décadas dando lugar a la existencia de "fragmentos educativos" caracterizados por el cierre social. Podría sostenerse que el sistema educativo argentino presenta condiciones de escolarización distintas entre escuelas y sectores dando lugar a la existencia de segmentación educativa (Velázquez, 2015).

El concepto de segmentación educativa contiene una discusión filosófica y los criterios para definirla y medirla varían de manera considerable. Podría definirse como la subdivisión de los sistemas educativos en escuelas o programas paralelos que difieren tanto en sus planes de estudio, como en los orígenes sociales de sus estudiantes o en circuitos educativos de distinta calidad (Ringer, 1992). En 
concordancia, Jaume (2013) sostiene que hay segmentación escolar cuando puede separarse a la población estudiantil entre establecimientos educativos de acuerdo a cierta/s característica/s generando circuitos marcadamente diferenciados.

Entonces, la definición de segmentación tiene anidada otra: la de circuito educativo. Tuñon y Alperin (2011) lo definen como un fenómeno que permite que los distintos grupos sociales que hacen uso del sistema educativo accedan a distintos niveles de educación formal, pero además, aún en caso de acceder a niveles de educación formal iguales acceden a niveles de conocimiento no equivalentes. A su vez, Braslavsky (1985) sostiene que los circuitos educativos son conformados por características que permiten identificarlos fácilmente como la condición socioeconómica del alumnado que asiste, el tipo de gestión, el perfil del personal que los conforma, etc. Puede sostenerse que la existencia de circuitos marcadamente diferentes es una condición necesaria para la presencia de segmentación educativa (Ball, Bowe y Gewirtz, 1995; Acosta, 2012), y da lugar a que los padres conformen estrategias para posicionar a sus hijos en los circuitos de mayor prestigio (Katzman, 2001; Verhoeven, 2013).

En general los circuitos constituyen grupos de diferente calidad educativa, entonces la movilidad de alumnos y centros de un circuito a otro es prácticamente nula (Tosoni y Nantel, 2010; Ibañez Martín, 2014). Cada tipo de centro atiende a un determinado perfil de alumnos, lo que llevará a la conformación de un determinismo social respecto a la calidad educativa a la que cada persona puede aspirar condicionada a que sus características personales coincidan con el perfil buscado. De ser así, los padres que poseen condiciones socioeconómicas poco favorables probablemente enviarán a sus niños -si lo hacen- a escuelas de baja calidad educativa, sometiéndolos a una enseñanza de menor nivel, con mayor riesgo de repitencia o abandono y a la obtención de credenciales educativas de menor valor que si hubieran accedido a otro tipo de centro (Solís, 2013). Finalmente, la existencia de circuitos educativos condiciona el fenómeno de movilidad y las expectativas de los individuos respecto a poder mejorar su posición en la jerarquía social (Ibañez Martin, 2014).

El fenómeno de segmentación educativa tiene diferentes expresiones, en función de cuál sea la variable que justifica su existencia. En particular, debido al interés de este trabajo, la condición socioeconómica del alumnado genera una disociación entre los establecimientos de gestión pública y los privados. Los circuitos atienden diferentes perfiles de alumnos y brindan una calidad educativa marcadamente diferente (Gasparini et al., 2010). En este sentido, es importante destacar que los circuitos no son internamente homogéneos y por ello se generan 
subcircuitos intersectoriales; si bien existe una relación entre los sectores de gestión y los circuitos educativos, tal relación no es lineal, en la medida que la segmentación se reproduce también al interior de ambos sectores (Pascual y Bottinelli, 2013). Es en este esquema que Llach (2006) reconoce la existencia de "escuelas pobres para pobres".

Entonces, la segmentación permite reconocer que la población estudiantil no se distribuye de manera homogénea entre los centros educativos y que la calidad que brindan los establecimientos tampoco lo es (Acidiácono et al. 2014; Bonal, 2015). El primer fenómeno podría resumirse en las ideas de Tiramonti (2004), al preguntarse ¿qué es lo que está segregado? ¿qué es materia de segregación? La lógica de pensamiento que surge por detrás de un fenómeno como la segregación educativa está estrechamente relacionada con la que conlleva el concepto de equidad.

La definición de segregar es "separar y marginar a una persona o a un grupo de personas por motivos sociales, políticos o culturales". En lo que respecta a la educación, puede darse segregación cuando la distribución de los alumnos es desigual entre los centros educativos por motivos económicos, sociales, culturales, de raza, género entre otros (Jenkins et al., 2008; Mancebón y Pérez-Ximénez, 2010). Tiramonti (2004) postula que la investigación del sistema educativo argentino da clara cuenta de ruptura de un campo de sentido compartido entre las instituciones y los individuos que circulan por ella. En sus palabras "hay un modo de constitución de lo institucional que genera la condición fragmentada".

La segregación educativa puede fundarse en diversas causas pero la que interesa, en torno al objetivo de investigación, es la fundamentada en el origen socioeconómico del alumno. La segregación por nivel socioeconómico implica que los alumnos más favorecidos asisten a establecimientos cuyo ambiente está provisto por mejores recursos y bajas probabilidades de interacción con niños y adolescentes de otro estrato social (Alcoba, 2015). Los individuos de bajo estrato social experimentan las condiciones inversas, se distribuyen en los centros de peores condiciones socioeconómicas, con peores recursos educativos y tienen bajas chances de interactuar con individuos de mejores estratos sociales.

Diversos estudios en países del mundo (Jenkins et al., 2008; Maroy 2008; Maroy y Van Zanten, 2009; Villa, Bonal y Verger, 2014) concluyen que los sistemas educativos presentan un grado de segregación moderado, que puede atribuirse a las políticas que han flexibilizado las condiciones de ingreso y liberado los mecanismo de elección de los padres e instituciones. 
Entonces, si existiera segregación socioeconómica del alumnado podría sostenerse que la distribución será desigual inter e intra-sectorial. En el primer caso, segregación pública/privada, un fenómeno que se ha visto favorecido por la condición institucional del sistema educativo argentino. En el año 1978 se comenzó con un proceso de descentralización de la provisión del servicio que tuvo una marcada tendencia economicista, en el cual se delegó a las provincias la responsabilidad pero no fue acompañado de un aumento de fondos (Formichella y Rojas, 2007). Dicha presión presupuestaria recayó sobre las provincias, deteriorando la calidad y equidad educativa (Morduchowicz, 2010).

Los estudios sobre la segmentación entre el sector privado y público para Argentina son escasos. Un estudio de Gasparini et al. (2010) tiene por objetivo la reconstitución de la segregación escolar argentina desde mediados de la década de los $80^{\prime}$. En el trabajo se utilizan distintas técnicas relativas a la estimación de segregación para comprobar su existencia y cómo ha evolucionado, usando encuestas de alta densidad poblacional para realizarlos ${ }^{1}$. Los autores sostienen que existe un aumento de la segregación educativa en función de la gestión escolar en los niveles de primario y secundario, intensificándose a nivel intersectorial en la última década y esperando el mismo comportamiento a corto plazo.

En la misma línea, Vázquez (2012) encuentra que en América Latina, y Argentina en particular, existe un alto nivel de segregación del alumnado basado en las condiciones socioeconómicas. A su vez, relacionado con la segregación intra-sectorial, sugiere que la segregación escolar por nivel socioeconómico al interior de las escuelas privadas es en general superior a la que ocurre dentro del agregado de escuelas públicas.

El trabajo de Jaume (2013), realiza un análisis empírico del nivel y evolución de la segregación escolar argentina entre escuelas públicas y privadas desde el año 1992 al 2010. Se utilizan las curvas de segregación percentílicas y micro descomposiciones ${ }^{2}$. Las conclusiones del trabajo son preocupantes, la segregación verifica un aumento que varía entre el $30 \%$ y $100 \%$ en función del índice y el nivel educativo analizados.

Adicionalmente, Arcidiácono et al (2014) realizan una investigación para América Latina y encuentra que la segregación escolar en Argentina ha aumentado

\footnotetext{
1 Para mayor detalle de las técnicas estadísticas y bases de datos utilizadas consultar: Gasparini, L., Jaume, D.; Serio, M.; y Vazquez, E. (2010).

2 Ambas técnicas serán detalladas en la sección metodológica del presente trabajo.
} 
en los últimos 20 años y se corresponde con un aumento en la desigualdad del ingreso (al igual que el resto de los países del cono Sur).

La privatización ha colaborado con un proceso de dualización socioeducativa más agudo que el verificado hasta la década del noventa (Rivas, Vera y Bezem, 2010). Los estratos más altos de la población fueron abandonando paulatinamente el sector público, percibiendo a la escolarización privada como una opción de mejor calidad, de posicionamiento social y de "resguardo" frente a interacciones con otras clases sociales. La dualización se agudizó con el aumento de acceso al nivel medio por parte de los estratos sociales más bajos, que antes eran excluidos (Fernández Cardoszo, 2011). Se verificó un crecimiento importante en la matriculación del nivel medio de los dos quintiles más bajos de la población, siguiendo estadísticas de SEDLAC: durante la década de los 90' la tasa neta de escolarización promedio de los dos primeros quintiles de la población rondaba en los 60 puntos porcentuales, mientras que en 2012 se verifica una tasa cercana al $82 \%$. A su vez, para el 2012 el $90 \%$ de la población en edad de asistir a la escuela media del primer quintil lo hacía a centros públicos y el $82 \%$ del decil subsiguiente. A partir de estos datos, puede sostenerse que los centros de gestión pública atienden a los sectores más desfavorecidos que no encuentran dentro de sus opciones factibles a la educación privada.

La migración de los sectores medios al sector privado es una evidencia que se verifica con mayor intensidad en la última década. Según el SEDLAC la tasa de escolarización neta para el nivel secundario en el sector público ha verificado una caída del 10 puntos porcentuales entre el 2003 y el 2011 para el segundo decil y de 6 puntos para el tercer decil, retornando a la tendencia ascendente en el 2012. Asimismo, existe un éxodo por parte de sectores menos favorecidos que también contemplan la educación privada como mecanismo de ruptura de la jerarquía social existente (Gamallo, 2011).

En lo que respecta a la segregación del alumnado dentro de cada circuito, se puede mencionar la existencia de dos circuitos internos dentro de la esfera privada: las escuelas de administración privada y las subvencionadas por el Estado. El funcionamiento de estos subcircuitos es diferente, en cuanto a administración, aceptación de alumnos, políticas de ingreso (Vázquez, 2012; Pascual y Bottinelli, 2013; Ibañez Martín, Delbianco y London, 2014).

En el sector público también se identifica segregación del alumnado, las escuelas públicas dependientes de las Universidades y las subvencionadas como las religiosas suelen tener un criterio de selección de alumnos diferente a las 
convencionales escuelas públicas, priorizando alumnos con mejores condiciones socioeconómicas y ciertos "conocimientos previos" al ingreso (Osorio, 2015). Por otro lado, la calidad educativa entre los colegios públicos suele ser distinta en función de su ubicación territorial y el entorno en el que están inmersas (Cohen, 2003); en palabras de Llach (2006) existen "escuelas pobres para pobres".

La otra vertiente de la segregación educativa, referenciada a la diferente calidad de servicios educativos que poseen las escuelas, se relaciona de manera directa con el perfil socioeconómico del alumnado (Ibañez Martín, Delbianco y London, 2014). A partir de su existencia puede hablarse de escuelas con alta o baja calidad educativa, las cuales no comparten ni traspasan alumnos entre sí (Gasparini et al, 2010).

La existencia de instituciones con diferentes calidades educativas conlleva un aumento y profundización de las diferencias de origen, perpetuando los determinismos sociales y la jerarquía existente en una sociedad. La caída generalizada de la calidad, tanto en las escuelas buenas como en las malas, implicó que el paso por el nivel primario y secundario sea visto como una condición necesaria para acceder a la universidad, perdiendo su importancia como motor de movilidad social (datos en páginas de OCDE, CAF). Se puede sostener que la segmentación educativa será más severa en aquellos sistemas educativos en los que la distribución de recursos materias, humanos, los sistemas de gobernanza y el clima en los establecimientos sea más desigual y tenga una relación con el perfil socioeconómico del alumnado (Cortés, 2008; Pipkin et al., 2014). Por ejemplo, el país A tendrá un sistema educativo más segmentado que el país B si las escuelas que atienden chicos pobres (en el primer país) tienen sólo un décimo de los recursos educativos y atraen al $80 \%$ de la población estudiantil; mientras que en el país B las escuelas pobres atienden la misma proporción del alumnado que las escuelas con mejor posición pero tienen una partida presupuestaria mayor que les permita compensar las diferencias de origen, por ejemplo dar la copa de leche.

La existencia del circuito educativo en función de la distribución de los recursos es un aspecto estudiado en la literatura especializada. La evidencia muestra que en los países en desarrollo los recursos son distribuidos en función del perfil socioeconómico de los alumnos, siendo más notorio en el nivel primario (OCDE, 2010). En América Latina existe una desigual distribución de los recursos que termina perjudicando a los sectores más desatendidos por la sociedad (Treviño et al., 2010). Particularmente en Argentina, los estudios comprueban la existencia del circuito donde la calidad de los recursos empeora en función del perfil socioeconómico que atienen de las instituciones (Llach, 2006; Falus y 
Goldberg, 2010). A su vez, la distribución de los insumos es desigual entre las escuelas privadas y públicas. Rivas, Vera y Bezem (2010) encuentran que en la provincia de Buenos Aires las escuelas privadas tenían un presupuesto anual por alumno mayor que las escuelas públicas y que dicha diferencia rondaba en el $40 \%$.

Concluyendo, podemos notar que la diferencia en la posesión de insumos educativos condiciona la calidad educativa que perciben los alumnos de distintos centros educativos: asistir a una institución privada o pública implica una desigual disposición de recursos. Toman protagonismo las diferencias de origen y la posición social en el condicionamiento respecto al acceso y calidad educativa (Lever 2012; Alcoba, 2013). Entonces, frente a la existencia de una desigual distribución de recursos educativos, las escuelas dejan de cumplir su rol de "alisador de caminos" $\mathrm{y}$ terminan perpetuando las diferencias iniciales.

\section{ESTUDIO EMPIRICO: ¿EL SISTEMA EDUCATIVO ARGENTINO VERIFICA SEGMENTACIÓN EN LA ÚLTIMA DÉCADA?}

\section{II.1 Modelo de decisión escolar}

Como ha sido mencionado anteriormente, la segregación educativa puede fundamentarse a partir de la presencia de diversos factores. En concordancia, Schelling (1978) menciona que la gente se segrega de diversas formas y bajo diferentes circunstancias. En el presente trabajo se estudiará, desde una perspectiva empírica, la segmentación del sistema educativo argentino fundamentada en la posición socioeconómica del alumnado.

En muchos casos la condición socioeconómica interfiere en la forma en la que los individuos deciden sobre los futuros educativos, dando lugar a un proceso de segregación basado en la elección individual (Jaume, 2013). Tal como menciona Córdoba (2011) muy a menudo, la composición social, la disciplina escolar o la distancia son criterios más centrales en las decisiones de elección escolar de las familias que otros elementos más directamente relacionados con la calidad educativa de las escuelas. En tal sentido, la segmentación escolar por nivel socioeconómico puede explicarse a partir de la decisión que toman las familias respecto a la educación de los niños. Podría suponerse que las familias maximizan su utilidad (dadas las preferencias) respecto a las opciones escolares de sus hijos, definidas como: no asistir, asistir a un colegio público ó privado. Así, las características familiares juegan un rol importante en el resultado del proceso de decisión. 
Siguiendo esa lógica de pensamiento, podría sostenerse que las familias se enfrentan a un proceso de decisión escalonado. En un primer momento, las familias deciden si insertar o no a sus hijos en el circuito educativo. En este momento el capital sociocultural y educativo de los padres cumple un rol fundamental, debido a que el valor asignado a la educación y la inversión que conlleva es fuertemente influenciado por la experiencia que los padres han tenido en el sistema educativo y luego de su paso por el mismo. En esta primer instancia, el nivel socioeconómico de la familia también tiene un fuerte impacto: las familias pobres enfrentan un mayor costo de oportunidad de la educación, enviar a un hijo a la escuela implica un recurso menos para generar ingresos en el hogar (Todaro y Smith, 2006). En la segunda etapa, en caso de que en el primer momento del juego se decida enviar a los niños a la escuela, las familias deberán decidir entre establecimiento público o privado. Aquí el nivel de ingresos familiar es un determinante esencial, es necesario un nivel determinado para afrontar las matrículas de los colegios privados (Jaume, 2013). A su vez, las expectativas o creencias respecto a la superioridad del sistema privado sobre el público en la generación de mejores credenciales educativas y oportunidades laborales, jugarán un rol central.

En otras palabras, la percepción que se tiene es que las opciones no están alineadas entre sí y, por el contrario, son excluyentes. Primero se enfrentan a las opciones asistir y no asistir; luego, en caso de asistir, se decide entre escuelas privadas o públicas. Bajo dicha concepción, se propone modelar las decisiones familiares a través de modelos Ordered Logit, que serán explicados en la siguiente sección.

Finalmente, dada la disponibilidad de datos y siguiendo la especificación de Jaume (2013), se tomaron en cuenta dos conjuntos de variables explicativas. Un vector de características del niño, compuesto por la edad y el sexo y, por otro lado, un vector de características familiares en el que se incorpora el logaritmo del ingreso per cápita familiar, la educación de los padres y el decil de ingresos en el que se posiciona la familia.

\section{II.2. Metodología y base de datos}

Tal como fue mencionado con anterioridad la elección familiar será representada por modelos logit ordenados y su versión generalizada, Ordered logit y generalized ordered logit respectivamente (Williams, 2006). La obtención de sus resultados tiene por objetivo el análisis de la segmentación educativa en Argentina durante la última década y sus determinantes. 


\section{II.2.1. Base de Datos}

Debido al interés de estudio, la segmentación dentro del sistema educativo argentino y la forma en que los alumnos se distribuyen entre escuelas privadas y públicas, los requerimientos sobre la base de datos son restrictivos. Se necesita disponer de información respecto a qué tipo de centro asiste cada alumno (público o privado), también sobre estrato económico al que pertenece cada persona o familia, por lo que se contará con el nivel de ingresos per cápita familiar y el decil de ingresos al que pertenece y, por último, es vital contar con información respecto a la experiencia educativa de los padres para evaluar su efecto sobre las decisiones escolares.

Los requisitos recién enumerados son cumplidos, en Argentina, únicamente por determinadas encuestas de hogares. Asimismo dado que el período temporal de interés es la última década, la encuesta que cuenta con mayor cantidad de observaciones y cumple con todos los requerimientos es la Encuesta Permanente de Hogares (EPH). El período analizado, del año 2004 al 2014, coincide con el cambio metodológico realizado por el Instituto Nacional de Estadísticas y Censos (INDEC) a partir del cual la EPH tomó su formato continuo. Los cambios se resumen en tres puntos: se rediseñaron los cuestionarios para captar mejor la situación del mercado laboral, mayor frecuencia en la exposición de la información y se rediseñó la muestra para obtener información continua (INDEC, 2003). Los dos últimos puntos permiten que la EPH sea una muestra compatible con los objetivos de investigación, haciendo innecesario empalmar información proveniente de diferentes fuentes ó tomar períodos temporales discontinuos.

La Encuesta Permanente de Hogares, es realizada por el INDEC y es de carácter urbano, dado que representa las dos terceras partes de la población nacional. Si bien la EPH es realizada desde el año 1974, donde se indagaba sobre el nivel de ingresos familiar e individual, sólo a partir del año 2003 comienza a considerar la asistencia escolar de los niños y la gestión del centro al que asisten. La incorporación sobre el tipo de centro coincide con el cambio en la modalidad de realización de la encuesta, empezando a realizarse cada trimestre. Sin embargo, cabe destacar que en el año 1998 se realizó un módulo especial de educación a nivel país, donde es posible identificar el tipo de establecimiento al que asiste cada individuo. Debido a la discontinuidad en el tema educativo, saltando desde 1998 al segundo semestre del 2003, se decidió evaluar el periodo comprendido por los años 2004 al 2013. Los análisis, en los modelos utilizados, serán realizados para todo el período temporal y también para los extremos. 
La población bajo estudio se remite a las personas de 5 a 19 años que asisten a un centro educativo, ya sea primario o secundario, o que han abandonado antes culminar con su formación en el año de referencia. A nivel individual son consideradas la edad y el sexo (que toma valor 1 en caso de ser varón).

Las características familiares tomadas en cuenta por nuestro modelo de decisión fueron: el nivel de ingreso per cápita familiar, que ha sido tomado en su versión logarítmica; el nivel educativo de los padres, tomando el nivel del padre que verifique la mayor cantidad de años de estudios completos; y por último, el decil de ingresos al que pertenece la familia. Respecto a esta última variable, se han estimado cuatro variantes. Por un lado, se tomó la variable provista por la base de datos, que considera todos los deciles. Por otro, con el fin de realizar un análisis más detallado se generaron otras tres variantes:

- Últimos deciles: que tomará valor 1 si la familia pertenece a los deciles 9 ó 10 , y cero si no pertenece.

- Primeros deciles: es una variable definida tal que tomará valor 1 si la familia pertenece a los dos primeros deciles de la distribución y cero en caso contrario.

- Escalas deciles: será igual a

1: si la familia pertenece a los dos primeros deciles.

2: si el hogar se ubica en los deciles 3 al 8 , inclusive.

3: si la familia se posiciona en los deciles 9 o 10 .

La desagregación de la variable encuentra justificación en la posibilidad de identificar un comportamiento diferente en función al decil en que se ubique la familia. En otras palabras, dicho procedimiento permitirá (en principio) estudiar si existe un comportamiento diferente entre las alumnos pobres y no pobres de la muestra, definidos en función de si pertenecen a los primeros ó últimos dos deciles de la distribución respectivamente. A su vez, la noción de agregar en grupos a los deciles se debe a que las diferencias no suelen notarse entre deciles continuos sino más bien entre bloques de deciles. En el caso particular de este estudio, el contexto en el cual se enfrentan a la decisión de asistencia no dista mucho entre el decil 1 y 2 , ó entre los deciles 9 y 10 (siendo que se controla por otras variables). Pero es claramente distinto entre personas de la cola inferior y superior de distribución de ingresos.

Finalmente, se optó por trabajar sólo con el primer y tercer trimestre de cada año. La elección se basa, fundamentalmente, en que en el primer trimestre del año comienza el año lectivo y se realizan las inscripciones de los alumnos a las escuelas; por su parte, en el tercer trimestre ya se ha superado la primera mitad 
del ciclo lectivo, por lo que permanecen en el sistema. A su vez, es apropiado considerar que el ingreso a comienzos de año a cierto tipo de centro se mantiene (en la mayoría de los casos) durante todo el período, mientras que los casos de abandono o cambio de escuela serían poco representativos en la muestra.

\section{II.2.2. Modelo Logit Ordenado y sus resultados}

Los modelos de regresión logística son utilizados para la estimación de problemas o situaciones en los que variables dependientes no son continuas, sino más bien variables dependientes dicotómicas o policotomas (Simon, 2004). El objetivo principal de los modelos de regresión logística, logit su término en inglés, es estimar la probabilidad de que un evento suceda dados los valores de las variables explicativas incorporadas (Liao, 1994). Los modelos logit se diferencian del modelo de probabilidad lineal ${ }^{3}$, debido a que suponen que el logaritmo de la razón de probabilidades está linealmente relacionado con las variables regresoras; mientras que el último supone la linealidad entre la regresada y las dependientes (Gujarati y Porter, 2009). Dado el objetivo de la presente investigación, es adecuado aplicar modelos de regresión logística que indiquen el efecto de las características familiares e individuales consideradas sobre la decisión escolar. Por ejemplo, se utilizaran modelos logísticos que nos indiquen cómo varía la probabilidad de asistir a un colegio privado frente al hecho de pertenecer a diferentes deciles de ingresos.

Como fue especificado, aquí se presentan tres categorías para la variable dependiente: No asistir, asistir a escuela pública ó establecimiento privado. Debido a que las opciones son excluyentes y pueden ordenarse, el primer conjunto de estimaciones corresponde a un modelo logit ordenado (una variante de los modelos de probabilidades proporcionales). La versión ordenada de los modelos logísticos supone que las variables respuesta tienen más de dos categorías y pueden ser jerarquizadas (Williams, 2006). En el marco de la investigación, las opciones a las que se enfrentan las familias cumplen con dicha característica: primero deben decidir si enviarán o no a los niños al sistema educativo, luego decidirán (en caso de enviarlos) entre centros privados o públicos ${ }^{4}$. El orden de las variables ha sido justificado por la proporción que representan del total de la muestra y por las restricciones que debe enfrentar la familia para poder superar cada categoría.

3 El modelo de probabilidad tiene numerosas crítica,s que escapan al objetivo de la investigación. Para mayor detalle se recomienda al lector revisar Gujarati (2009).

4 La jerarquización se realiza considerando cuán operativa es la restricción presupuestaria en cada estadío de la desición. 
Los coeficientes del modelo nos dirán, por ejemplo, si la probabilidad de no asistir está positiva o negativamente relacionada con el hecho de ser mujer, de pertenecer a una familia de ingresos bajos o tener padres con un bajo nivel educativo. La interpretación de los modelos de regresión logística, en todas sus variantes, tienen cierta dificultad debido a que los estimadores indican cómo varía la razón de probabilidades ante un cambio en las variables explicativas (Gujarati y Porter, 2009). Sin embargo, en el presente estudio no se busca una cuantificación exacta del cambio en la probabilidad de que un niño asista a una escuela pública o privada, sino más bien cómo afectan ciertas características a la decisión que tomen sus familias respecto a su futuro educativo.

A su vez, en la especificación, los coeficientes que acompañan a las variables dependientes no varían entre las categorías. Dicha condición respecto a los coeficientes es conocido como el supuesto de probabilidades proporcionales (Wolfe y Gould, 1998). Contrariamente, los términos independientes dependen de la categoría por lo que las regresiones tendrán diferente ordenada al origen pero igual pendiente generando regresiones paralelas (Long y Freese, 2006).

Una vez establecidas, no exhaustivamente ${ }^{5}$, las características de los modelos logísticos y logísticos ordenados es pertinente explicitar cuáles son las categorías definidas para la variable respuesta y el número asociado a las mismas. Las opciones pueden ordenarse de la siguiente manera:

0 . No asistir a la escuela.

1. Asistir a establecimiento público.

2. Asistir a escuela privada.

Primero se estimó el modelo para todo el periodo temporal con cuatro especificaciones distintas, en función de variable indicadora de decil de ingreso utilizada: deccfr (variable proporcionada por la base de datos), últimos deciles, primeros deciles y escalas deciles. En la tabla a continuación se presentarán, por columnas, los resultados hallados en el orden antes mencionado.

$\mathrm{Al}$ analizar los resultados arriba expuestos, habría evidencia respecto a que los niños que pertenecen a familias ubicadas en los primeros deciles de la distribución tienen menores probabilidades de asistir al colegio que aquellos que se ubican del tercer decil en adelante. A su vez, pertenecer a familias ubicadas

5 Para un mayor detalle sobre los modelos Logit Ordenados, se recomienda al lector revisar: Williams, R. (2006). 
en la proporción de la población más rica aumenta las probabilidades de asistir al sistema educativo y de hacerlo a una institución de gestión privada. El efecto positivo sobre la asistencia a centros privados es mayor para los últimos dos deciles de la distribución que para los ubicados entre los extremos. Bajo dicho resultado, habría evidencia a favor de la hipótesis de segmentación educativa fundada en las condiciones socioeconómicas del alumno.

Tabla II.2.1. Modelo Order Logit: Período 2004-2014

\begin{tabular}{|c|c|c|c|c|}
\hline Order Logit & DECCFR (1) & Últimos Deciles & Primeros Deciles (2) & Escalas Deciles (4) \\
\hline \multirow{2}{*}{$\begin{array}{l}\text { Decil de } \\
\text { Ingresos }\end{array}$} & 0.2229258 & 1.233621 & -1.007511 & 0.9306804 \\
\hline & $(0.0012138)^{* * *}$ & $(0.012378)^{* * *}$ & $(0.0062747)^{* * *}$ & $(0.005287)^{* * *}$ \\
\hline \multirow[t]{2}{*}{ Edad } & 0.175343 & -0.1509339 & -0.1569929 & -0.158988 \\
\hline & $(0.0056509)^{* * *}$ & $(0.000714) * * *$ & $(0.0007175)^{* * *}$ & $(0.0007216)^{* * *}$ \\
\hline \multirow[t]{2}{*}{ Sexo } & 0.2137967 & 0.1837967 & 0.1917514 & 0.1940961 \\
\hline & $(0.0058974)^{* * *}$ & $(0.0056788)^{* * *}$ & $(0.0056977)^{* * *}$ & $(0.0057187)^{* * *}$ \\
\hline \multirow{2}{*}{$\begin{array}{l}\text { Educación } \\
\text { Padres }\end{array}$} & 0.0487901 & 0.0703784 & 0.0615164 & 0.0574327 \\
\hline & $(0.0004128)^{* * *}$ & $(0.0004127)^{* * *}$ & $(0.0004159)^{* * *}$ & $(0.0004192)^{* * *}$ \\
\hline
\end{tabular}

$*, * *$ y ***: significativos al 10,5 y $1 \%$ respectivamente. Desvíos estándar entre paréntesis.

Fuente: Estimaciones propias en Base a EPH.

A su vez, es interesante destacar que la probabilidad de asistir a la escuela disminuye cuando aumenta la edad del niño. La relación negativa se mantiene respecto a la posibilidad de asistir y hacerlo a un centro privado de educación. Dicho resultado concuerda con la evolución de la asistencia al sistema educativo verificada en Argentina, descripta anteriormente. Se da la universalización en el nivel primario pero persisten las dificultades en el nivel secundario de educación, captando el fenómeno de deserción que persiste en el nivel medio. Sumado a que la obligatoriedad 
del nivel secundario sólo es establecido en la ley n ${ }^{\circ} 26.206$ en el año 2006, por lo que su efecto se evidencia sólo en la segunda mitad del período temporal analizado.

Asimismo, podría encontrarse cierto efecto de género en la segregación educativa de Argentina dado que es más probable que un niño asista a la escuela respecto a que lo haga una niña. Dicho aspecto puede visualizarse en la relación positiva que encuentra la variable sexo con las regresadas.

Finalmente, el nivel educativo de los padres tiene relación positiva con las categorías de la variable dependiente. Cuanto mayor sea la formación educativa del los padres más importancia le darán a la educación de sus hijos y las probabilidades de asistencia aumentarían. A su vez, podría pensarse que padres con mayor educación tienen mejores condiciones para tomar la decisión escolar modelizada. Padres con mayor educación suelen tener mejores trabajos, verificar un mejor nivel socioeconómico y, por ende, una mayor propensión a enviar a sus hijos a escuelas privadas (Cruces et. al., 2011; Jaume, 2013).

A su vez, fueron estimados modelos logit ordenados para los extremos del periodo temporal considerado. Es decir, se estimaron las 4 especificaciones para el primer y tercer trimestre del 2004 y 2014. El objetivo de dicha estimación es ver si el comportamiento de la variable dependiente respecto de las explicativas ha cambiado con el paso de una década; sin embargo, los resultados anteriormente expuestos se mantienen en las estimaciones.

Ahora bien, como fue mencionado, los modelos ordered logit suponen proporcionalidad de las probabilidades respecto a las categorías de la variable regresada (Williams, 2006). Dicho supuesto es la fuente de críticas más importante que poseen estos modelos. La restricción que impone la suposición de regresiones paralelas es superada por la utilización de diversas variantes de los modelos de regresión logística, como la utilizada en la próxima sección. Sin embargo, las soluciones comúnmente utilizadas requieren de la estimación de mayor número parámetros que los estrictamente necesarios, poniendo en peligro los grados de libertad (op. cit.).

Luego de realizar cada una de las estimaciones, se realizó el test de Brant (1990) que evalúa la validez del supuesto de regresiones paralelas. En todos los casos, el test arrojó el rechazo de la hipótesis nula: existencia de probabilidades proporcionales. A partir de ello, se decidió estimar la versión generalizada de logit ordenados porque no requieren del supuesto de $\beta$ constantes (Williams, 2006). 


\section{II.2.3. Modelo Logit Ordenado Generalizado (Generalized Ordered Logit)}

La versión generalizada del modelo logit ordenado persigue -conceptualmente- el mismo objetivo: estudiar las variaciones en la razón de probabilidades de un evento posible ante cambios en las variables regresoras, dado que la variable regresada puede tomar distintos valores jerarquizados.

La distinción entre logit ordenado y la versión generalizada se funda en el supuesto de regresiones paralelas, mencionado párrafos arriba. Los modelos generalized ordered logit (su nombre en inglés) no suponen independencia de los coeficientes que acompañan a las variables regresoras respecto de las categorías, como lo hace su versión no generalizada. Es decir, los modelos son menos restrictivos que el ordered logit debido a que contemplan la posibilidad de que los coeficientes $\beta$ varíen en función de la categoría de la variable regresada (Williams, 2006). A su vez, son modelos más parsimoniosos e interpretables que los logísticos multinomiales, como el utilizado por Jaume (2013).

Sencillamente, la idea que está por detrás del ordered logit generalizado es analizar cómo se comporta la probabilidad de que un individuo se traslade de la categoría 1 a la 2, y sucesivamente, en función de las variables explicativas incorporadas al modelo. En otras palabras, se reconoce que cada variable contribuye de manera diferente a pasar de una categoría a la inmediata, por lo que no habría restricciones paralelas. Las variables explicativas utilizadas, las especificaciones del modelo y el análisis temporal coinciden con lo realizado en la sección anterior. Los resultados del generalized ordered logit para el periodo temporal completo pueden observarse en la Tabla II.2.3.1.

Los resultados demuestran que pertenecer a los primeros deciles de la distribución de ingresos, ser niña, tener padres con escasa educación y avanzar en edad afectan negativamente a la probabilidad de sobrepasar la categoría "no asistir". Los resultados se mantienen, con mayor severidad, en las probabilidades de asistir a escuela privada. En base a lo expuesto, puede sostenerse que en la última década el sistema educativo argentino ha evidenciado un proceso de segregación en base a las condiciones socioeconómicas de los alumnos y el nivel educativo del hogar al que pertenece. La presencia de efectos negativos sobre la decisión familiar basados en cuestiones económicas y culturales ha reforzado la segmentación educativa y, consecuentemente, la existencia de inequidad en la calidad educativa que reciben los alumnos pertenecientes a diferentes realidades socioculturales. 


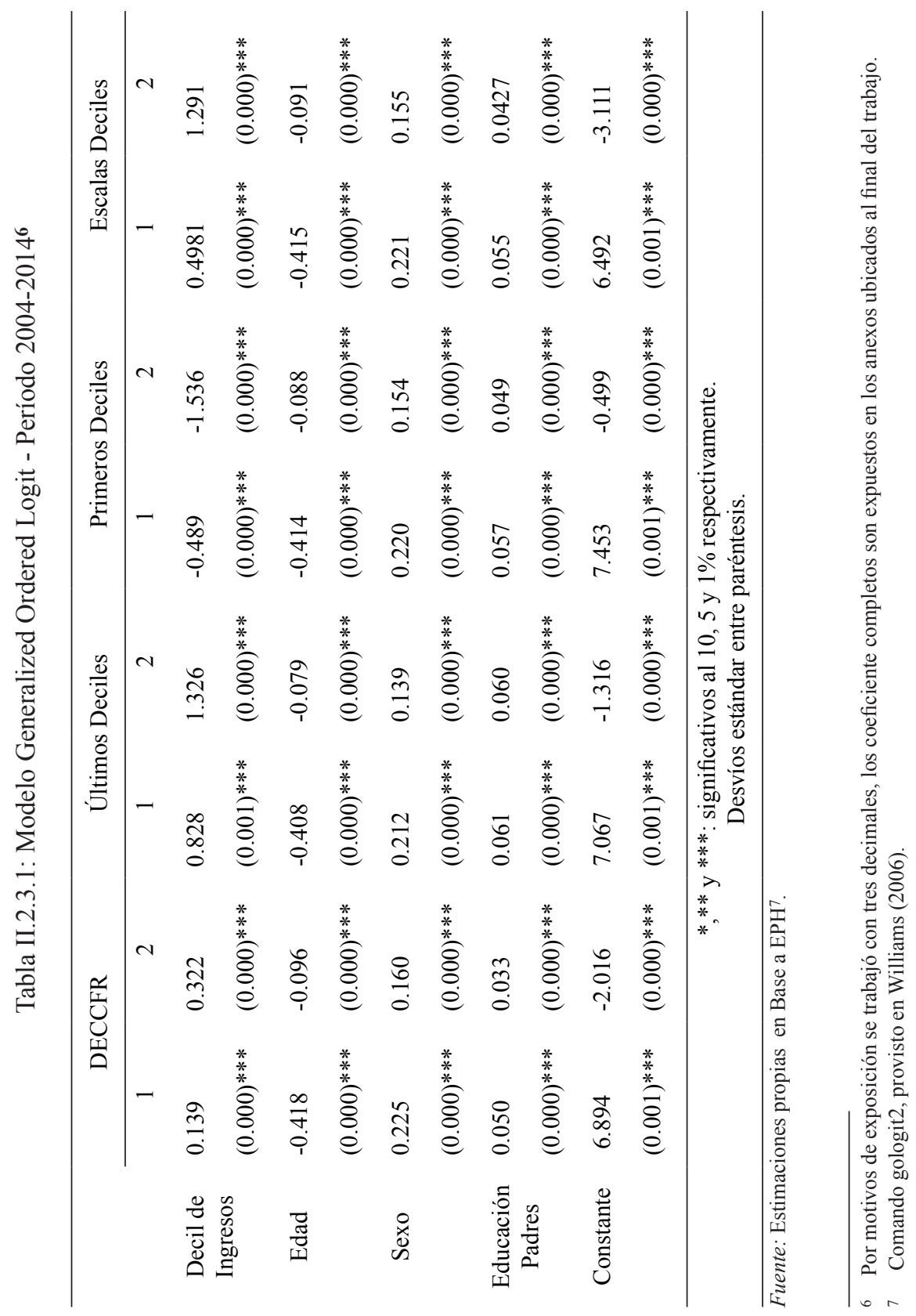


A su vez, ubicarse del tercer decil de ingresos en adelante aumenta las probabilidades de asistir a escuelas públicas y privadas. Sin embargo, el hecho de ubicarse en los últimos dos aumenta las probabilidades de asistir a ambos tipos de instituciones con mayor impacto que pertenecer a los deciles del medio de la distribución (i,e. esto puede verse en la diferencia de las estimaciones obtenidas para las variables escalas deciles y últimos deciles). Los resultados concuerdan con diversos estudios que postulan que la asistencia a escuelas privadas no se relaciona, actualmente, con personas de alto poder adquisitivo y que la migración hacia el sector privado también se presenta en familias de ingresos medios y bajos.

Nuevamente, podría sostenerse que existe cierto "efecto género" en la distribución de los niños dentro del sistema educativo. Los varones presentan mayores probabilidades que las niñas de sobrepasar la no asistencia y de hacerlo a escuelas privadas. La justificación podría encontrarse en el rol que cumplen las niñas en los hogares más pobres, siendo una ayuda importante en el cuidado de los más pequeños y en los quehaceres domésticos (Silva, 2006).

En cuanto a la edad, como fue mencionado antes, su aumento tiene un efecto negativo sobre las probabilidades de asistir a la escuela. Nuevamente, al igual que los resultados presentados en la sección anterior, las conclusiones van en concordancia con la evolución que ha tenido el acceso al sistema educativo de nuestro país. Se ha dado universalización del nivel primario y se ha mejorado en el acceso del nivel secundario, sin embargo los problemas de abandono y rezago son mayores en el último (Formichella, 2010).

Finalmente, el nivel educativo de los padres tiene el signo previsto por la economía de la educación. Padres con mayor formación tienen mayor tendencia a enviar a sus hijos a la escuela y hacerlo a establecimientos privados. Nuevamente, como postulan Gvirtz et. al (2007) y Larrañaga (2010) padres con más educación buscan que sus hijos logren credenciales educativas de valor, que no interactúen con niños de otros estratos sociales y valoran más la inversión que la educación conlleva.

Los resultados de los extremos temporales, 2004 y 2014 por separado, muestran la misma relación entre las variables explicativas y las categorías de la variable dependiente. El impacto de la edad, el sexo, el nivel de ingresos y el nivel educativo de los padres no ha cambiado con el paso de la última década.

Entonces, en concordancia con las ideas de Gómez Schettini (2007) puede sostenerse que existen dos tipos de familias, un grupo de familias cautivas, obligadas a optar por la oferta educativa pública la cual es aceptada pasivamente 
debido a la escasez de recursos económicos y culturales. Existe un segundo grupo de familias que posee información y capital cultural y social que les permite buscar otras alternativas públicas o privadas.

\section{CONCLUSIONES}

La disponibilidad de la base de datos de la Encuesta Permanente de Hogares realizada por el Instituto de Estadísticas y Censos de Argentina ha permitido la estimación de diversos modelos que representan la decisión familiar respecto al futuro educativo de los niños.

Los distintos modelos estimados, optando por el Generalized Ordered Logit debido a que sus supuestos son menos restrictivos que el Ordered Logit tradicional, permiten realizar algunas reflexiones respecto a la situación del sistema educativo argentino en la última década. Sin dudas, los centros educativos atienden diferente alumnado en función de la clase social a la que pertenecen y el ambiente socioeducativo en el que están inmersos.

El decil de ingresos en el que se ubica la unidad familiar tiene relación positiva con la probabilidad de asistir al sistema y de hacerlo a centros privados. Es decir, a medida que se avanza en el decil de la distribución aumentan las probabilidades de que una familia decida enviar a sus niños a centros privados. Asimismo, padres con mayor educación tienden a fomentar la asistencia al sistema educativo y su formación -al igual que su posición económica- afecta positivamente las probabilidades de avanzar en las categorías de la variable dependiente del modelo logístico ordenado.

Por tanto, puede sostenerse que el sistema argentino tiene problemas en la dimensión interna de la equidad educativa. Los niños con peores condiciones socioeconómicas enfrentan probabilidades mayores de inasistencia y, en caso de asistir, lo harán con mayores chances a establecimientos públicos. La noción de Llach et al (2006) de escuelas pobres para pobres sería apoyada por la evidencia del estudio empírico realizado.

Concluyendo, el principal objetivo ha sido estudiar la segmentación educativa en Argentina, a partir del nivel socioeconómico del alumnado. Se ha tomado en cuenta el proceso de decisión que realizan las familias y estimado distintos modelos econométricos para estudiar el impacto de las características familiares e individuales en la decisión. Los resultados encontrados van en 
concordancia con los obtenidos en estudios empíricos anteriores (Gasparini et al, 2012; Jaume, 2013). El aporte distintivo es la lógica de decisión familiar, donde las familias no se enfrentan a todas las alternativas, dependiendo fuertemente de la capacidad monetaria y de la experiencia que hayan tenido los padres en su paso por el sistema educativo.

Las distintas categorías de la variable dependiente y la jerarquización han sido esquematizada a partir de modelos ordered logit y generalized ordered logit. Las estimaciones son consistentes entre sí, los efectos de las variables explicativas se mantienen. Sin embargo, debido al rechazo de la hipótesis nula en los tests de restricciones aplicados a las estimaciones logísticas ordenadas resulta pertinente la aplicación de la versión generalizada de los modelos.

\section{REFERENCIAS BIBLIOGRÁFICAS}

Arcidiácono. M., Cruces, G., Gasparini, L., Jaume, D., Serio M. y Vazquez, E. (2014). La segregación escolar público-privado en América Latina. CEDLAS, Documento de Trabajo N ${ }^{\circ} 167$. Recuperado de file://C:/Users/ Maria/Downloads/doc_cedlas167.pdf

Acosta, F. (2012). La escuela secundaria argentina en perspectiva histórica y comparada: modelos institucionales y desgranamiento durante el siglo XX. Cuadernos de História da Educação, 11 (1), 131-144.

Alcoba, M. (2015). La dimensión social del logro individual: Desigualdad de oportunidades educativas y laborales en Argentina. (Tesis doctoral). FLACSO México.

Alcoba, M. (2013). Desigualdad de oportunidades en el espacio educativo. Argentina, 1950-2007. Revista Latinoamericana de Población, 7(12), 5-31.

Aranda, J. C. F. (2013). Procesos Segregatorios y Segmentación Educativa. Sintesis, (3). Recuperada de http://www.revistas.unc.edu.ar/index.php/ sintesis/article/viewFile/8235/9115

Arneson, R.J. (1989). Equality and equal opportunity for welfare. Philosophical studies, 56(1), 77-93.

Aristizábal, A. B., Aguilar, J. F. L., \& Walker, M. (2010). La educación superior desde el enfoque de capacidades: una propuesta para el debate. Revista electrónica interuniversitaria de formación del profesorado, 13(3), 123-131.

Gewirtz, S., Ball, S. J., \& Bowe, R. (1995). Markets, choice, and equity in education. Buckingham: Open University Press.

Blanco, R. (2006). La inclusión en educación: una cuestión de justicia y de igualdad. Revista Sinéctica, 29, p19. 
Bolívar, A. (2011). Justicia social y equidad escolar. Una revisión actual. Revista internacional de educación para la justicia social. 1(1), p. 9-45.

Bonal, X. (2015). Crisis, educación y desigualdad: una cuestión de paradojas. Investigar em Educação, 1(3), 23-32.

Brant, R. (1990). Assessing proportionality in the proportional odds model for ordinal logistic regression. Biometrics, 46 (4), 1171-1178.

Braslavsky, C. (1985). La discriminación educativa en Argentina. Buenos Aire: FLACSO, Grupo Editor Latinoamericano.

Calero, J. (1999). Indicadores de equidad interna y externa en la educación superior. Metodologías y una aplicación al caso español. Ponencia presentada en el Seminario Indicadores universitarios: tendencias y experiencias internacionales. Ministerio de Cultura y Educación, Secretaría de Políticas Universitarias, Buenos Aires.

Calero, J. (1999). Indicadores de equidad interna y externa en la educación superior. Metodologías y una aplicación al caso español. Ponencia presentada en el Seminario Indicadores universitarios: tendencias y experiencias internacionales, Ministerio de Cultura y Educación, Secretaría de Políticas Universitarias, Buenos Aires. Recuperado de http://descargas-api.educ.ar/ sitios/educar/recursos/ver?id=93331

Cameron, A. C., \& Trivedi, P. K. (2005). Microeconometrics: methods and applications. Estados Unidos: Cambridge university press.

Cervini, R. (2009). Comparando la inequidad en los logros escolares de la educación primaria y secundaria de Argentina: un estudio multinivel comparativo. REICE. Revista Electrónica Iberoamericana sobre Calidad, Eficacia y Cambio en Educación, 7 (1), 6-21.

Cohen, G. A. (1989). "On the currency of egalitarian justice". Ethics, 906944. Recuperado de http://www.utexas.edu/law/journals/tlr/sources/ Volume\%2092/Issue\%206/Rogers/Rogers.fn060.Cohen.OntheCurrency. pdf

Cohen, E., Martínez, R., Donoso, P. y Aguirre, F. (2003). Localización de infraestructura educativa para localidades urbanas de la Provincia de Buenos Aires (Vol. 79). Santiago de Chile: United Nations Publications.

Córdoba, C. (2011). Elección de escuela en Chile: La propuesta neoliberal a examen. (Tesis doctoral). Universidad Complutense de Madrid.

Cortés, F. (diciembre, 2008). Desigualdad, educación media y el mundo del trabajo: una mirada sobre la relación educación-trabajo en establecimientos educativos de nivel medio, de la especialidad técnica de la ciudad de Buenos Aires. Trabajo presentado en las $V$ Jornadas de Sociología de la UNLP. Universidad Nacional de La Plata. Facultad de Humanidades y Ciencias de la Educación. Departamento de Sociología, La Plata, Argentina. 
Cruces, G., Garcia-Domenech, C., \& Gasparini, L. (2012). Inequality in Education. Evidence for Latin America. CEDLAS, Documento de Trabajo $\mathrm{N}^{\circ}$ 135. Recuperado de http://cedlas.econo.unlp.edu.ar/esp/areas-detrabajo.php?idA=8

DiNIECE (2010a). "Una mirada sobre la escuela III. 40 indicadores. Ministerio de Educación Nacional”. [en línea] http://repositorio.educacion.gov. ar:8080/dspace/handle/123456789/96484

DiNIECE (2010b). "PISA 2009: Resumen Ejecutivo Argentina”. [en línea] http:// diniece.me.gov.ar/index.php?option $=$ com_content\&task=category\&sectio nid $=3 \& \mathrm{id}=15 \&$ Itemid $=26$

Dupriez, V. (2010). "Methods of grouping learners at schools". Fundamentals of Educational Planning 93. Paris: IIPE-UNESCO.

Duru-bellat, M. \& Van Zanten, A. (2001). Sociologie de l'école, Paris: Armand Colin.

Enguita, M. F. (2013). La igualdad, la equidad y otras complejidades de la justicia educativa. Revista Portuguesa de Educação, 26(2), p, 205-224.

Falus, L. \& Golberg, M. (2010). Recursos, instalaciones y servicios básicos en las escuelas de América Latina. Otra forma que asume la desigualdad educativa. SITEAL. Documento de Trabajo: Cuaderno 07

Fernández, T., \& Cardozo, S. (2011). Tipos de desigualdad educativa, regímenes de bienestar e instituciones en América Latina: un abordaje con base en PISA 2009. Páginas de educación, 4 (1), 33-55.

Formichella, M. M. (2011). Análisis del concepto de equidad educativa a la luz del enfoque de las capacidades de Amartya Sen. Revista Educación, 35 (1), 1-36.

Formichella, M. (2010). Educación y desarrollo: análisis desde la perspectiva de la equidad educativa interna y del mercado laboral. (Tesis Doctoral), Universidad Nacional del Sur.

Formichella, M. \& Ibañez Martín, M.M (2014) Gender and education inequality: an analysis of Argentina. Regional and Sectoral Economic Studies/ Estudios Económicos Regionales y Sectoriales (RSES). Vol 14 PP- 195-210.

Formichella, M. \& Rojas, M. (2007). El proceso de descentralización de la educación en la Argentina. Un caso: La Provincia de Buenos Aires. En A. Goetschel (comp.): Perspectivas de la educación en América Latina (pp. 167188). Ecuador: Ed. FLACSO Ecuador y Ministerio de Cultura de Ecuador.

Gamallo, G. (2011). Mercantilización del bienestar. Hogares pobres y escuelas privadas. Revista de Instituciones, Ideas y Mercados, 55, p. 189-233.

García-Huidobro, J. (2005). La equidad en educación como bien democrático y de desarrollo. Presentación realizada en la Reunión del Comité Intergubernamental del PREALC. Santiago de Chile 6 y 7 de diciembre. 
Gasparini, L., Jaume, D., Serio, M., \& Vazquez, E. (noviembre, 2010). La segregación escolar en Argentina. Reconstruyendo la evidencia. Anales de la XLV Reunión Anual de la Asociación Argentina de Economía Política. Bs. As. Recuperado de http://www.aaep.org.ar

Gómez Schettini, M. (2007). La elección de los no elegidos: los sectores de bajos ingresos ante la elección de la escuela en la zona sur de la Ciudad de Buenos Aires. Escuelas Y Familias Problemas De Diversidad Cultural Y Justicia Social, 101-126.

Gorard, S. \& Smith, E. (2004). "An international comparison of equity in education systems". Comparative Education, 40 (1), 15-28.

Gujarati, D. \& Porter, D. (2009). Econometría (5a ed.). México: Mc Graw Hill.

Gvirtz, S., Grinberg, S., \& Abregú, V. (2007). La educación ayer, hoy y mañana. Buenos Aires: Aique.

Ibañez Martin, M., Delbianco, F. \& London, S. (noviembre, 2014). Segmentación educativa en Argentina durante la última década: sus determinantes desde los modelos Generalized Ordered Logit. Anales de XLIX Reunión Anual de la Asociación Argentina de Economía Política. Recuperado de http://www. aaep.org.ar/anales/works/works2014/ibanez_delbianco.pdf

Ibáñez Martín, M. M. (2015). Segmentación e inequidad educativa en Argentina: su relación con la movilidad social. Tesis de Maestría en Economía, Universidad Nacional del Sur. Disponible en: http://repositoriodigital.uns. edu.ar/handle/123456789/2374

Ibañez Martin, M., \& Formichella, MM. (noviembre, 2014). Género y logros educativos: un análisis a partir de un modelo multinivel trivariado. Anales de XLIX Reunión Anual de la Asociación Argentina de Economía Política. Recuperado de http://www.aaep.org.ar/anales/works/works2014/ibanez. pdf

Instituto Nacional de Estadísticas y Censos (INDEC). (2003). Encuesta Permanente de Hogares: Cambios Metodológicos. Recuperado de http://www.indec. mecon.ar/ftp/cuadros/sociedad/Gacetilla_EPHContinua.pdf

Jaume, D. (2013). Un estudio sobre el incremento de la segregación escolar argentina. CEDLAS, Documento de Trabajo $\mathrm{N}^{\circ} 143$. Recuperado de http:// cedlas.econo.unlp.edu.ar/esp/areas-de-trabajo.php?idA=8

Jenkins, S.; Micklewright, J. \& Schnepf, S. (2008). Social segregation in secondary schools: how does England compare with other countries?, Oxford Review of Education, 34 (1), 21-37.

Katzman, R. (2001). Seducidos y abandonados: el aislamiento social de los pobres urbanos. Revista de la CEPAL, 75, 171-189. 
Krüger, N. (2012). Equidad Educativa Interna y Externa en Argentina: un Análisis para las Últimas Décadas. Tesis Doctoral en Economía. Universidad Nacional del Sur.

Latapí, P. (1993). "Reflexiones sobre la justicia en la educación". Revista Latinoamericana de Estudios Educativos, vol. XXIII. núm. 2, pp. 9-41.

Lever, L. V. (2012). Tres problemas prioritarios que urge resolver en la educación media superior. Perfiles Educativos, 34, 170-175.

Larrañaga, O. (2010). Las nuevas políticas de protección social en perspectiva histórica. Documento de Trabajo, 4. http://www.ministeriodesarrollosocial. gob.cl/btca/txtcompleto/Larranaga_nvaspolitprotecsocial.pdf

Llach, J. (2006). El desafio de la equidad educativa. Ed. Granica.

Liao, T.F. (1994). Interpreting Probability Models: Logit, Probit and Other Generalized Models. Estados Unidos: Sage University Papers.

López, N. (2005). La educación en América Latina, entre el cambio social y la inercia institucional. Revista galega de economía: Publicación Interdisciplinar da Facultade de Ciencias Económicas e Empresariais, 14(1), 203-222.

Lopez, N. (2006). Equidad educativa y desigualdad social. Lugar de publicación: Ed. IIPE-UNESCO.

Loewe, D. (2009). El enfoque en las capacidades y las demandas por derechos culturales. Signos Filosóficos, 11(21), 103-146.

Long, J. \& Freese, J. (2006). Regression Models for Categorical Dependent Variables Using Stata (2a ed.). Estados Unidos: College Station, TX: Stata Press.

Mancebón, M. \& Pérez-Ximénez de Embún, D. (2007). Conciertos educativos y selección académica y social del alumnado. Hacienda Pública Españolal Revista de Economía Pública, 180 (1), p. 77-108.

Marchesi, Á., Blanco, R., Hernández, L., \& Educativas, M. (2014). Avances y desafíos de la educación inclusiva en Iberoamérica. Madrid: FUNDACIONMAPFRE.

Maroy, C. (2008). ¿Por qué y cómo regular el mercado educativo?, Profesorado: Revista de Curriculum y Formación del Profesorado, 12 (2), 1-11. Recuperado de http://redalyc.uaemex.mx/src/inicio/ArtPdfRed. jsp? $\mathrm{iCve}=56712202$

Maroy, C. \& Van Zanten, A. (2009). Regulation and competition among schools in six European localities, Sociologie du travail, 51(1), pp. 67-79.

Morduchowicz, A. (2010). El federalismo fiscal-educativo argentino. En Andrade Oliveria et al:: Políticas educativas y territorios modelos de articulación entre niveles de gobierno (pp. 225-260). Bs. As.: IIPE-Unesco.

Morresi, S., Donnini, N. y Cerioni, L. (2009). Evaluación de la calidad en la educación superior. Su abordaje en países seleccionados. III Congreso Nacio- 
nal y II Encuentro Internacional de Estudios Comparados en Educación. Buenos Aires: Saece.

Muñoz Izquierdo, C (2009). ¿Cómo puede la educación contribuir a la movilidad social? Resultados de cuatro décadas de investigación sobre la calidad y los efectos socioeconómicos de la educación. México: Universidad Iberoamericana.

OCDE (2010). PISA 2009 Results: Overcoming Social Background. Equity in Learning Opportunities and Outcomes. (Informe de investigación Vol. IV). Recuperado de http://dx.doi.org/10.1787/9789264091559-en

Osorio, E. O. (2015). Un estudio de los mecanismos de selección y regulación de la matrícula en tres escuelas públicas de la Ciudad Autónoma de Buenos Aires. (Tesis Doctoral). Recuperada de http://repositorio.flacsoandes.edu. ec/bitstream/10469/6864/2/TFLACSO-2014EOO.pdf

Pascual, L., \& Bottinelli, L. (2013). El debate sobre el crecimiento reciente de la educación privada. (Documento de la DiNIECE No. 11). Recuperado de

http://repositorio.educacion.gov.ar:8080/dspace/bitstream/handle

/123456789/109841/educa11.pdf?sequence $=1$

Pipkin, D., Zibecchi, C., Larrondo, M., Federico, H., Guadalupe, L., \& Fernanda, M. (diciembre, 2014). Escuela media, desigualdad educativa y enseñanza de las Ciencias Sociales: notas para un diseño de investigación. VIII Jornadas de Sociología de la UNLP. Recuperado de http://jornadassociologia.fahce. unlp.edu.ar/viii-jornadas-2014/PonMesa7PIPKIN.pdf

Roemer, J. E. (1955). Equality and responsibility, Boston Review Forum, Social Equality and Personal Responsibility, XX(2). Disponible en http:// bostonreview.net/dreader/series/equality.html

Ringer, F. K. (1992). La segmentación en los modernos sistemas educativos europeos: el caso de la educación secundaria en Francia entre 1865 y 1920. El desarrollo del sistema educativo moderno: cambio estructural y reproducción social 1870-1920 (pp. 87-130). España: Servicio de Publicaciones.

Rivas, A.; Vera, A. \& Bezem, P. (2010). Radiografía de la educación argentina. Buenos Aires: Fundación CIPPEC; Fundación Arcor; Fundación Roberto Noble.

Rivero, J. (1999). Políticas Educativas de Equidad e Igualdad de Oportunidades. Presentación realizada en el III Seminario para Altos Directivos de las Administraciones Educativas de los países Iberoamericanos. La Habana.

Sen, A. (1979) "Equality of what?" The tanner lecture of human values. Stanford University.

Schelling, T. (1978). Micromotives and Macrobehavior. New York: Norton. 
Silva, M. J. (2006). Niñas, niños y adolescentes: los riesgos de un trabajo invisible para el propio hogar. Santiago de Chile: Bureau régional de Organización Internacional del Trabajo (OIT).

Silva-Laya, M. (2012). Equidad en la Educación Superior en México: La necesidad de un Nuevo concepto y Nuevas Políticas. Archivos Analíticos de Políticas Educativas Vol, 20(4), 2.

SITEAL (Marzo, 2008). Caras nuevas: cambios en la composición socioeconómica de los estudiantes del nivel medio en la región Recuperado del sitio de internet del Sistema de Información de Tendencias en América Latina, Sección Datos Destacados: http://www.siteal.iipe-oei.org/datos_destacados/238/nuevos-alumnos-en-el-nivel-medio

Solís, P. (2013). Desigualdad vertical y horizontal en las transiciones educativas en México. Estudios Sociológicos, 31, número extraordinario, 63-95

Tiramonti, G. (2004). La configuración fragmentada del sistema educativo argentino, Cuaderno de Pedagogía Rosario, 7(12), p 33-46.

Todaro, M., \& Smith, S. C. (2006). Development economics. Estados Unidos: Pearson.

Tosoni, M. M., \& Natel, A. (2010). Elegí esta escuela primaria para mi hijo. Las elecciones educativas de las familias de sectores populares. VI Jornadas de Sociología de la UNLP. Recuperado de http://www.aacademica.com/000027/22/368.pdf

Treviño, E., Valdés, H., Castro, M., Costilla, R., Pardo, C. \& Donoso, F. (2010). Factores asociados al logro cognitivo de los estudiantes de América Latina y El Caribe. Santiago: OREALC/UNESCO Santiago \& LLECE.

Tuñón, I., \& Halperin, V. (diciembre, 2009). Desigualdad social en la calidad de la oferta educativa y percepción de la calidad educativa. En I Congreso de Sociología de la Provincia De Buenos Aires. Recuperado de: http://www. uca.edu.ar/uca/common/grupo68/files/MT29_-_TU--N-HALPERIN.pdf

Vazquez, E. (2012). Segregación escolar por nivel socioeconómico. Midiendo el fenómeno y explorando sus determinantes. CEDLAS. Documento de Trabajo $\mathrm{N}^{\circ}$ 128. Recuperado de: file:///C:/Users/Maria/Downloads/doc_cedlas128.pdf

Velázquez, G. A. (2015). Calidad de vida y pobreza en la Argentina (2010). Aproximación a escala provincial. Journal de Ciencias Sociales, 3 (4), 4-18.

Verhoeven, M. (2013). Desigualdades múltiples, carreras escolares y pruebas en sistemas educativos postmasificación: Los efectos de la segmentación educativa en la construcción del sujeto. Propuesta educativa, 2 (40), 87-98.

Villa, A. Z., Bonal, X., \& Verger, A. (2014). Mercados educativos y segmentación de la oferta escolar: efectos sobre las desigualdades educativas en Chile. Témpora: Revista de historia y sociología de la educación, (17), 11-30. 
Viscardi, M. L. (2014). La política social educativa en la Argentina en perspectiva histórica: nuevos desafíos. Trabajo presentado en las $I X$ Jornadas de investigación, docencia, extensión y ejercicio profesional: "Transformaciones sociales, políticas públicas y conflictos emergentes en la sociedad argentina contemporánea".Facultad de Trabajo Social UNLP, La Plata, Argentina.

Williams, R. (2006). Generalized Ordered Logit/ Partial Proportional Odds Models for Ordinal Dependent Variables. The Stata Journal 6 (1), 58-82. A prepublication version is available at http://www.nd.edu/ rwilliam/gologit2/ gologit2.pdf .

Wolfe, R. \& Gould, W. (1998). An approximate likelihood-ratio test for ordinal response models, Stata Technical Bulletin 42, 24-27. Reimpreso en Stata Technical Bulletin Reprints, vol. 7, pp. 199-204. College Statio, TX: Stata Press

\section{PAGINAS WEB CONSULTADAS}

OECD (2014). La movilidad educativa se hace más lenta en el mundo industrializado, dice la OCDE. Recuperado de

http://www.oecd.org/centrodemexico/medios/la-movilidad-educativa-sehace-mas-lenta-en-el-mundo-industrializado-dice-la-ocde.htm

CAF (s.f.). Educación para la movilidad social: oportunidades en América Latina. Recuperado de http://www.caf.com/media/3093/Cap3.Educacionparalamovilida dsocial.pdf

(C) 2015 por los autores; licencia otorgada a la Revista Estudios Económicos. Este artículo es de acceso abierto y distribuido bajo los términos y condiciones de una licencia Atribución-No Comercial 3.0 Unported (CC BY-NC 3.0) de Creative Commons. Para ver una copia de esta licencia, visite http://creativecommons.org/ licenses/by-nc/3.0/ 


\title{
EMPLEO Y DESEMPLEO EN BAHIA BLANCA EN LOS AÑOS 2003-2013
}

\author{
Gustavo Burachik*
}

recibido: abril 2015 - aceptado: junio 2015

\section{INTRODUCCIÓN}

En este trabajo se analiza un período de 10 años de mediciones trimestrales de la Encuesta Permanente de Hogares Continua (EPH) del INDEC comprendidas entre el tercer trimestre de 2003 y el cuarto de 2013. Dado que estas estimaciones contienen un error estadístico apreciable ${ }^{1}$, el estudio se limita a señalar las principales tendencias en la evolución de las tasas laborales básicas de desocupación, empleo y actividad; no se formulan hipótesis explicativas respecto de variaciones cuantitativas menores, de corto plazo o referidas a los niveles de las variables para los cuales el error muestral resulta necesariamente más elevado.

La tabla 1 presenta las mencionadas tasas del aglomerado Bahía BlancaCerri $(\mathrm{ABBC})$ y las que corresponden a diversas desagregaciones de interés.

Se han tomado los cuartos trimestres de 2003, 2007 y 2013 para delimitar dos grandes períodos de la evolución económica nacional; el lustro 2003/7 durante el cual la economía nacional salió de la larga crisis iniciada en 1998 y el período 2008/2013, en que la mayor parte de los indicadores económicos, laborales y sociales dejó de mostrar progresos significativos.

El crecimiento del PBI per cápita se redujo de 7,8\% anual en 2003/7 a 2\% en 2008/13 y la desocupación, que en el primer período de cuatro años disminuyó en 6 puntos porcentuales (pp) sólo bajó otros 2 pp en el sexenio 2008/13. Nuestro

* Departamento de Economía (UNS) - Instituto de Investigaciones Económicas y Sociales del Sur (CONICET - UNS). Correo electrónico: burachik@criba.edu.ar.

1 Por ejemplo, el coeficiente de variación de la tasa de desocupación del aglomerado Bahía BlancaCerri en el IV- 2013 fue 16,3\%. Así, los límites del intervalo de $90 \%$ de confianza fueron 6,3 y $11 \%$. 
objetivo es estudiar la evolución de la situación laboral en el ABBC en estas dos etapas y entre los años extremos de la década 2003/13.

Tabla 1. Tasas de actividad, empleo y desocupación. Aglomerado Bahía Blanca-Cerri. Cuarto trimestre de cada año. En \%

\begin{tabular}{|c|c|c|c|c|c|c|c|c|c|}
\hline & \multicolumn{3}{|c|}{$\mathbf{T A}^{1}$} & \multicolumn{3}{|c|}{$\mathbf{T E}^{2}$} & \multicolumn{3}{|c|}{$\mathbf{T D}^{\mathbf{3}}$} \\
\hline & 2003 & 2007 & 2013 & 2003 & 2007 & 2013 & 2003 & 2007 & 2013 \\
\hline Aglomerado & 47 & 44 & 48 & 40 & 40 & 44 & 15 & 9 & 9 \\
\hline Varones & 58 & 56 & 58 & 49 & 51 & 56 & 15 & 9 & 5 \\
\hline Mujeres & 39 & 31 & 39 & 33 & 28 & 34 & 15 & 11 & 14 \\
\hline Jefes de hogar & 73 & 69 & 68 & 63 & 63 & 66 & 13 & 9 & 3 \\
\hline Cónyuges & 53 & 39 & 55 & 47 & 36 & 46 & 11 & 6 & 17 \\
\hline Hijos & 24 & 27 & 22 & 19 & 24 & 19 & 22 & 11 & 15 \\
\hline Edad 18-24 & 67 & 47 & 49 & 53 & 45 & 36 & 21 & 174 & 26 \\
\hline Edad 25-30 & 81 & 86 & 85 & 73 & 68 & 75 & 11 & 21 & 11 \\
\hline Edad 31-55 & 83 & 79 & 84 & 74 & 73 & 79 & 11 & 7 & 5 \\
\hline Edad 56-65 & 57 & 53 & 68 & 43 & 45 & 67 & 25 & 15 & 3 \\
\hline N. E. bajo 5 & 45 & 40 & 44 & 36 & 34 & 39 & 21 & 14 & 12 \\
\hline N. E. medio ${ }^{6}$ & 68 & 56 & 59 & 60 & 52 & 55 & 11 & 7 & 7 \\
\hline N. E. superior 7 & 95 & 88 & 84 & 91 & 83 & 78 & 4 & 5 & 7 \\
\hline $\begin{array}{l}\text { Tasa de actividad } \\
2 \text { Tasa de empleo = } \\
3 \text { Tasa de desocupac } \\
\text { El número de deso } \\
\text { En su lugar emple } \\
\text { a altas tasas. } \\
\text { Secundaria incomp } \\
\text { Secundario comple } \\
\text { Estudios superiore }\end{array}$ & $\begin{array}{l}\text { blación } \\
\text { ación } / \mathrm{p} \\
=\text { desoc } \\
\text { dos de } \\
\text { s el de I } \\
\text { o meno } \\
\text { estudio } \\
\text { mpletos. }\end{array}$ & $\begin{array}{l}\text { conómi } \\
\text { blación } \\
\text { pación/ } \\
\text { sta edad } \\
\text {-2008, r } \\
\text { superio }\end{array}$ & $\begin{array}{l}\text { amente } \\
\text { total. } \\
\text { oblació } \\
\text { en el IV } \\
\text { present } \\
\text { es inco }\end{array}$ & $\begin{array}{l}\text { activa/p } \\
\text { econór } \\
2007 \text { co } \\
\text { tivo del } \\
\text { apletos. }\end{array}$ & $\begin{array}{l}\text { icament } \\
\text { tenido e } \\
\text { período }\end{array}$ & $\begin{array}{l}\text { total. } \\
\text { e activa. } \\
\text { n la base } \\
\text { en que l }\end{array}$ & $\begin{array}{l}\text { de micr } \\
\text { econom }\end{array}$ & $\begin{array}{l}\text { datos es } \\
\text { ía deja }\end{array}$ & $\begin{array}{l}\text { erróneo. } \\
\text { e crecer }\end{array}$ \\
\hline
\end{tabular}

Fuente: EPH (INDEC). 


\section{EVOLUCION POR PERIODOS}

\section{I.1. Carácter acotado de la recuperación del empleo}

(a) Incidencia de ta y te en la evolución de la td

La disminución de la tasa de desocupación del ABBC de 15\% a 9\% de la población activa constituye un reflejo de la nueva etapa económica que se desarrolló en 2003/7. Como se muestra en la tabla 2, la evolución de la tasa de desocupación de los 31 aglomerados urbanos (31AU) que releva la EPH fue muy similar (de $14 \%$ a $8 \%$ ).

Tabla 2. Tasas de actividad, empleo y desocupación.

Total 31 aglomerados urbanos. Cuarto trimestre de cada año. En \%

\begin{tabular}{|c|c|c|c|c|c|c|c|c|c|}
\hline & \multicolumn{3}{|c|}{ TA } & \multicolumn{3}{|c|}{$\mathrm{TE}$} & \multicolumn{3}{|c|}{$\mathrm{TD}$} \\
\hline & 2003 & 2007 & 2013 & 2003 & 2007 & 2013 & 2003 & 2007 & 2013 \\
\hline Total aglomerados & 46 & 46 & 46 & 40 & 42 & 43 & 14 & 8 & 6 \\
\hline Varones & 55 & 55 & 54 & 48 & 52 & 51 & 12 & 6 & 6 \\
\hline Mujeres & 38 & 37 & 37 & 32 & 33 & 35 & 17 & 10 & 8 \\
\hline Jefes de hogar & 75 & 73 & 70 & 69 & 69 & 68 & 8 & 4 & 3 \\
\hline Cónyuges & 50 & 50 & 54 & 44 & 47 & 51 & 13 & 7 & 5 \\
\hline Hijos & 28 & 28 & 26 & 21 & 24 & 23 & 27 & 13 & 13 \\
\hline Edad 18-24 & 63 & 58 & 53 & 44 & 48 & 44 & 30 & 16 & 17 \\
\hline Edad 25-30 & 82 & 79 & 79 & 70 & 73 & 74 & 14 & 7 & 7 \\
\hline Edad 31-55 & 81 & 79 & 81 & 73 & 75 & 78 & 9 & 5 & 4 \\
\hline Edad 56-65 & 59 & 59 & 58 & 52 & 56 & 57 & 13 & 5 & 3 \\
\hline
\end{tabular}

Fuente: EPH (INDEC) 
Fue diferente, sin embargo, el proceso subyacente; la caída de la tasa de desocupación local se originó en una contracción de la tasa de actividad con una tasa de empleo constante mientras que a nivel de los $31 \mathrm{AU}$ fue exactamente al revés.

En el segundo subperíodo, entre 2007 y 2013, la tasa de desocupación del $\mathrm{ABBC}$ dejó de disminuir. En estos años la tasa de empleo por fin se incrementó pero el incremento contemporáneo de la tasa de actividad impidió que esto se tradujera en una nueva baja de la tasa de desocupación. A nivel de los 31AU, en cambio, se repitió lo ocurrido en el período anterior (aunque con menor intensidad); nueva disminución de la tasa de desocupación como resultado de un nuevo aumento de la tasa de empleo, con una tasa de actividad constante.

(b) Grado de difusión de la caída de la td y del aumento de la te

Ahora nos interesa verificar el alcance de la disminución de la tasa de desocupación y/o del aumento de la tasa de empleo entre diversos subgrupos de trabajadores. La tabla 1 muestra una desagregación de las tasas básicas según género, edad, rol en el hogar y nivel educativo para el ABBC.

Esta desagregación ofrece elementos de interés para el análisis de la situación de los diversos segmentos que componen a la fuerza de trabajo. Por un lado, permite observar el comportamiento diferenciado de su núcleo central compuesto por los varones adultos "jefes de hogar" respecto de los llamados "trabajadores secundarios", es decir, los cónyuges e hijos. El análisis por tramos de edad, por otro lado, da una pauta del dinamismo del sistema económico para asimilar y brindar oportunidades de ingreso y capacitación a las nuevas generaciones de trabajadores.

Este análisis ofrece un resultado interesante. La caída de la tasa de desocupación fue un fenómeno generalizado en 2003/7 entre los diversos subgrupos de trabajadores. En 2007/13, en cambio, la tasa de desocupación sólo siguió bajando entre los trabajadores varones, jefes de hogar, mayores de 25 años. En otros sectores (mujeres, jóvenes, personas con estudios universitarios completos), por el contrario, volvió a elevarse.

El incremento de la tasa de empleo luego de la larga crisis económica de 1998/2002, en cambio, se fue difundiendo al pasar del primer subperíodo al segundo. En 2003/7 la tasa de empleo sólo aumentó entre los hijos varones mientras que disminuyó entre las mujeres y para los trabajadores menores de 
56 años. En estos últimos sectores la disminución de la tasa de desocupación reposó íntegramente en una disminución de la oferta de trabajadores. En el lustro siguiente, en cambio, el aumento de la tasa de empleo se difundió al conjunto de los sectores de la fuerza de trabajo. Solo que contemporáneamente, como ya se indicó, se produjo un aumento de la tasa de actividad que impidió que la tasa de desocupación continuara bajando.

Resulta de interés la comparación entre esta evolución y la de los 31AU. También en este caso la tasa de desocupación dejó de disminuir al pasar de un período al siguiente en varios sectores (varones, hijos, trabajadores de hasta 30 años). La diferencia residió en el comportamiento de la tasa de empleo; a nivel de los $31 \mathrm{AU}$ en el primer período se incrementó de un modo generalizado mientras que en el segundo sólo siguió aumentando en subsectores específicos (mujeres, cónyuges, mayores de 24) y a contraerse en otros como varones, jefes, hijos de ambos sexos y trabajadores de 18-24 años.

En suma, en el ABBC la disminución de la tasa de desempleo se estancó en 2008/13 porque la tasa de actividad, que se redujo en 2003/7, comenzó a elevarse. A nivel de los $31 \mathrm{AU}$, en cambio, el ritmo de caída de la tasa de desocupación se desaceleró al moderarse y adquirir un carácter más acotado el aumento de la tasa de empleo.

\section{I.2. Obstáculos a la disminución de la desocupación}

Se arriba así a un segundo elemento que surge con claridad de los datos. Se trata de que de aquellas categorías en que la evolución del empleo resultó relativamente desfavorable, emerge una fuerza que mantiene la tasa de desocupación del $\mathrm{ABBC}$ en un nivel elevado tanto en términos históricos ${ }^{2} \operatorname{como}$ respecto a los demás aglomerados urbanos. En el análisis de los factores que influyen sobre la tasa de desocupación resulta útil considerar la siguiente relación entre las tasas laborales básicas ${ }^{3}$ :

$$
\text { Tasa de desocupación } \equiv 1-\frac{\text { Tasa de ocupación }}{\text { Tasa de actividad }}
$$

2 Las mediciones de los primeros dos años de la EPH puntual para el aglomerado ABBC en 1985/7 fluctuaron en \pm 2 pp en torno de una media de $5 \%$.

3 Ver definiciones de estas tasas al pie de la tabla 1. 
Así, la evolución de la tasa de desocupación en un período se puede expresar, alternativamente, en términos de una combinación dada de cambios en las tasas de actividad y empleo. Algunas relaciones son directas e intuitivas (para una tasa de actividad dada, la tasa de desocupación disminuye cuando se reduce la de ocupación). Otras lo son menos; la tasa de desocupación puede subir aunque la tasa de ocupación se incremente, si dicho aumento resulta inferior al de la tasa de actividad.

Por otra parte, al analizar las tasas básicas a nivel de los diversos subgrupos de trabajadores surge una dimensión adicional; aún cuando un subsector dado de la fuerza de trabajo experimente una disminución de su tasa de desocupación seguirá ejerciendo una presión al alza de la tasa del aglomerado si su nivel sigue superando el promedio de este último.

Dos sectores particulares de la fuerza de trabajo, tanto en el ABBC como en los 31AU, muestran una situación laboral relativamente desfavorable; las trabajadoras mujeres y los jóvenes. Son estos segmentos, en ambos casos, los que mantienen la tasa de desocupación en niveles relativamente elevados y son también los que explican la mayor desocupación del ABBC con respecto al total de los aglomerados urbanos del país. En otras palabras, la situación en general desfavorable de mujeres y jóvenes se presenta con particular agudeza en el ABBC.

\section{(a) Mujeres}

En ambos períodos las mujeres mostraron una tasa de desocupación relativamente elevada en el ABBC. En 2003/7 esto se vio reflejado en la alta desocupación de los hijos (fue elevada para los hijos de ambos sexos) y en 2008/13 también en la de los cónyuges ${ }^{4}$.

La desfavorable evolución de la ocupación femenina resulta notable. En 2003/7 la tasa de desocupación de las mujeres se redujo, pero menos que la de los varones. Además, esta reducción no fue el resultado de un aumento de la tasa de empleo (que, de hecho, se contrajo) sino de una brusca disminución de la tasa de actividad. En los años 2008/13, aunque la tasa de empleo de las mujeres experimentó un cierto aumento, la tasa de actividad subió mucho más y por ello la de desocupación no sólo no continuó con el descenso iniciado en los años previos sino que retomó la tendencia ascendente.

4 Otros datos revelan que en el período se aceleró la formación de nuevos hogares de modo que es esperable que el grupo de hijas mujeres desocupadas en 2003/7 figure como cónyuges desocupadas en el período siguiente. 
En el gráfico siguiente, la presión ascendente ejercida por la desocupación femenina se manifiesta a través de valores del índice superiores a la unidad. Como se puede ver, dicha presión estuvo presente de un modo casi permanente, excepto por un breve período en 2010/11.

Dos rasgos parecen caracterizar el comportamiento de la tasa de desocupación femenina del aglomerado. Uno, su nivel fluctúa sistemáticamente por encima de la media. Dos, también sistemáticamente las tasas de ocupación y desocupación femeninas varían en igual sentido debido a las fuertes variaciones de la tasa de actividad; en el primer subperíodo la fuerte contracción de la tasa de actividad permitió que cayera la desocupación pese a la caída de la ocupación y en el siguiente hizo que la tasa de desocupación se incrementara pese al simultáneo incremento de la tasa de ocupación.

Gráfico 1. Tasa de desocupación mujeres/tasa de desocupación varones

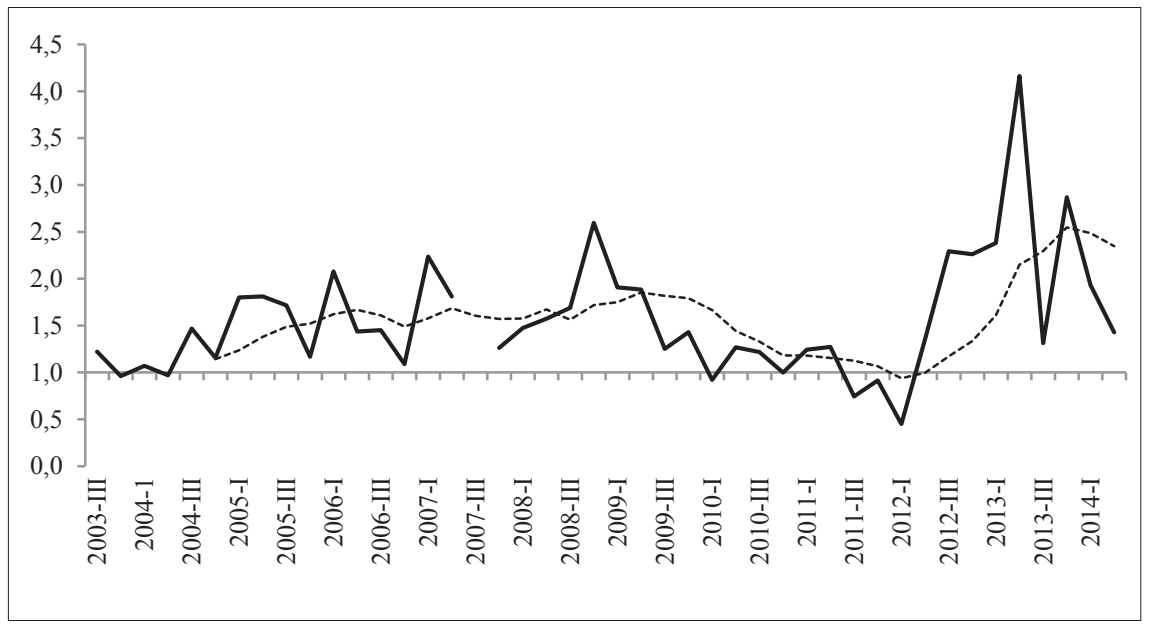

Fuente: EPH (INDEC)

En otras palabras, en 2003/7 muchas mujeres abandonaron o perdieron sus puestos de trabajo y muchas dejaron de buscar; aún así siguió siendo elevada la proporción de las que continuaron solicitando empleo sin encontrarlo. En 2007/13 tuvo lugar un cierto aumento en la disponibilidad de puestos de trabajo para las mujeres pero la cantidad de interesadas que acudió al mercado excedió a las vacantes dando lugar a un nuevo incremento de la tasa de desocupación. La comparecencia masiva de mujeres en busca de empleo (de ingresos adicionales) 
en 2008/13 en un contexto de estancamiento o declinación de los salarios reales contribuyó al deterioro relativo de la situación laboral de las mujeres ya que la tasa de desocupación de los varones continuó disminuyendo.

Hay aquí otro contraste con el total de los aglomerados. Mientras que la tasa de desocupación masculina del $\mathrm{ABBC}$ ha tendido a converger con la de los 31AU, lo contrario ha ocurrido con la femenina que resultó en 2013 6,5 pp (puntos porcentuales) más alta. En IV-2013 la tasa de desocupación de las mujeres bahienses resultó $86 \%$ más alta que en los $31 \mathrm{AU}$.

(b) Jóvenes

También los trabajadores jóvenes muestran una evolución laboral problemática. La tasa de desocupación de los trabajadores de hasta 30 años se ha mantenido sistemáticamente por encima del promedio en el $\mathrm{ABBC}$ al igual que en los 31AU. En ninguno de los dos ámbitos se han notado mejoras sustanciales entre 2008 y 2013.

La elevada tasa de desocupación relativa de los jóvenes se aprecia también cuando se considera el rol en el hogar de los trabajadores; la de los "hijos" (ambos sexos) resulta siempre superior al promedio, tanto en $\mathrm{ABBC}$ como en los $31 \mathrm{AU}$. A decir verdad, la tasa de desocupación de los jefes de hogar del $\mathrm{ABBC}$ ha tendido a converger con su similar de los $31 \mathrm{AU}$; pero las de los demás miembros del hogar (cónyuges e hijos) resultan más elevadas en el ABBC.

En el siguiente gráfico, valores del índice superiores a la unidad muestran niveles de desocupación juvenil más altas que el promedio. Para los dos segmentos de edad seleccionados la tasa de desocupación de los jóvenes resulta sistemáticamente más alta que la de los trabajadores de 31 a 55 años.

En suma, la razón de que la tasa de desocupación del ABBC resulte más elevada que a nivel nacional reside en la mayor desocupación relativa de la fuerza de trabajo "adicional" o "secundaria", es decir, mujeres y jóvenes.

(c) Nivel educativo

Se dispone para el ABBC de información sobre el nivel educativo máximo alcanzado por los trabajadores. La tabla 1 muestra 3 agrupamientos; bajo, medio y alto. Como se observa en la tabla 1, los trabajadores con "bajo" nivel educativo 
presentan sistemáticamente una tasa de desocupación más elevada que la del promedio del $\mathrm{ABBC}$.

En el subperíodo 2003/7, la tasa de desocupación de este grupo de trabajadores se redujo debido a la disminución de su tasa de actividad ya que la tasa de empleo disminuyó. En el subperíodo siguiente la desocupación se mantuvo elevada al producirse una fuerte expansión de la oferta. El gráfico que sigue muestra la elevada tasa de desocupación de los trabajadores de nivel bajo respecto del promedio en la mayor parte de la última década.

Gráfico 2. Tasa de desocupación trabajadores jóvenes/tasa de desocupación trabajadores entre 31 y 55 años

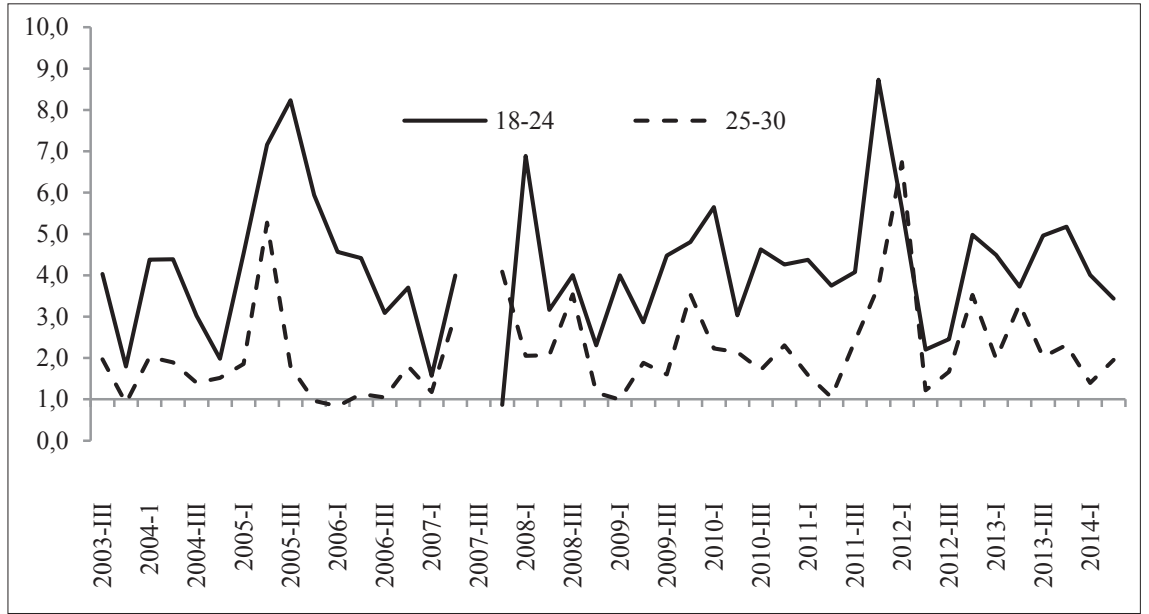

Fuente: EPH (INDEC)

Por último, el gráfico que sigue muestra las diferencias de las tasas de desocupación de la fuerza de trabajo de nivel educativo medio y bajo respecto de las que cuenta con estudios universitarios completos. El gráfico muestra una erosión de la ventaja de inserción laboral vinculada con el nivel educativo. Como se ve, en las primeras mediciones de 2003 la tasa de desocupación de los trabajadores de menor formación era unos 10 puntos porcentuales más alta que la de los universitarios. Esta brecha tendió a estrecharse luego. En los últimos años los trabajadores de nivel educativo "alto" ya sólo muestran una menor desocupación respecto de los trabajadores de nivel educativo bajo; la que tenían hace una década respecto de los de nivel medio parece haber desaparecido. 
Gráfico 3. Tasa de desocupación trabajadores con nivel educativo bajo/tasa de desocupación promedio

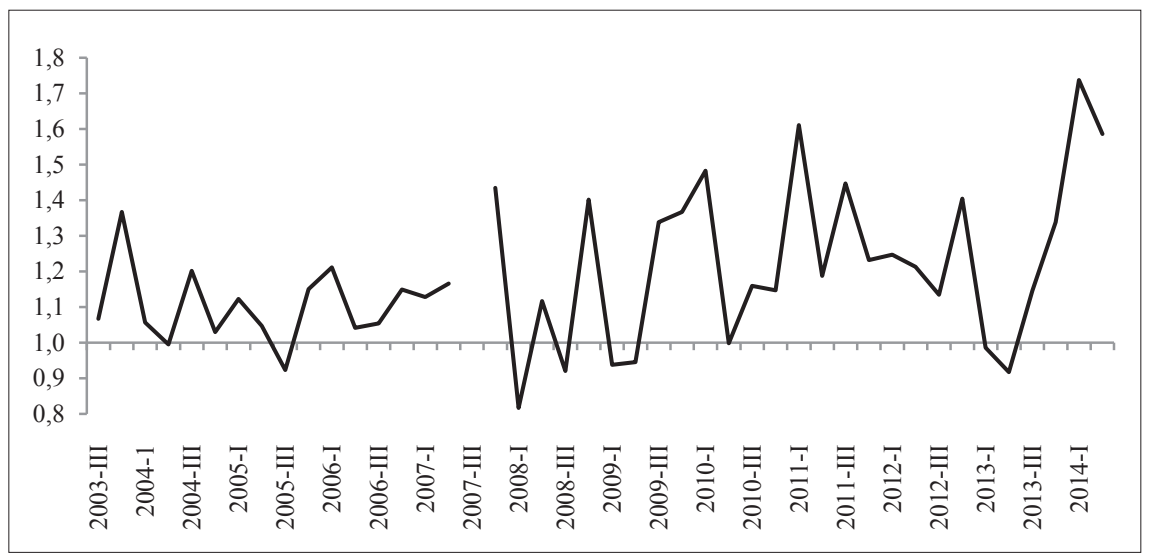

Fuente: EPH (INDEC)

Gráfico 4. Diferencias tasas de desocupación niveles educativos "medio" y "bajo" respecto de "alto" (en puntos porcentuales). Promedio móvil cuatro trimestres

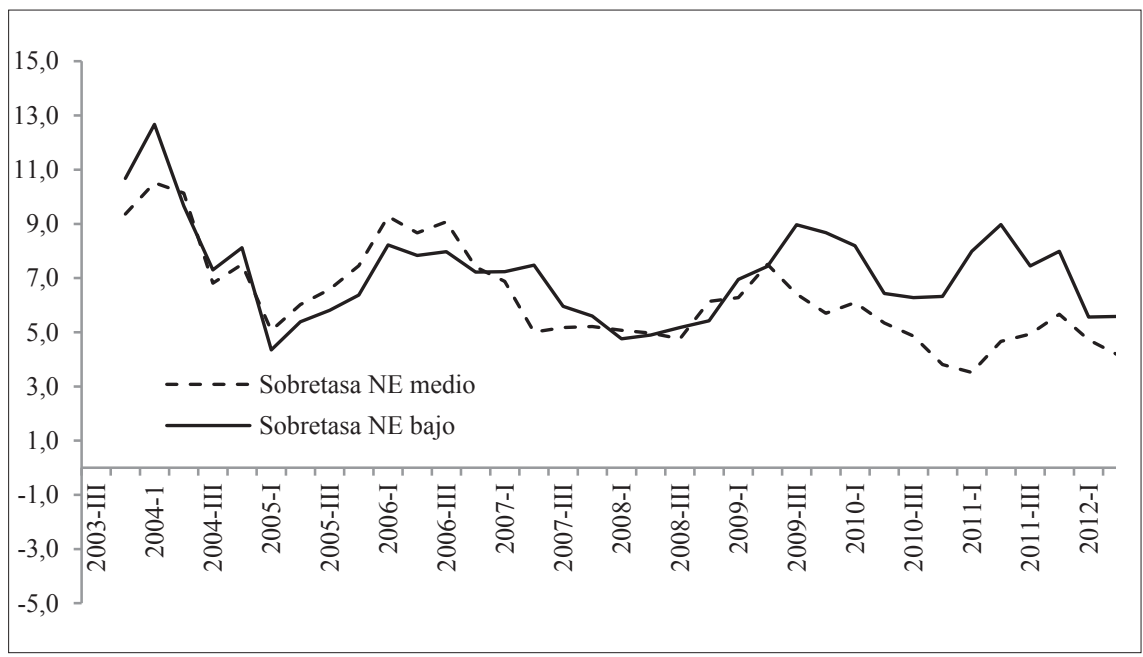

Fuente: EPH (INDEC) 
I.3. Desocupación más elevada que en el resto de los aglomerados urbanos

La tasa de desocupación del ABBC ya era más elevada que en los $31 \mathrm{AU}$ en 2003 y la brecha ha tendido a crecer; en IV-2013 fue 40\% más alta que la del total urbano. Las causas de esta diferencia, además, fueron cambiando. En los primeros años era explicada por la tasa de desocupación relativamente alta de los varones y jefes de hogar pero luego ésta convergió con el promedio urbano nacional y la divergencia pasó a residir en la fuerza de trabajo "secundaria", básicamente mujeres y personas que en el hogar son identificados como cónyuges o hijos. Esto se debió, a su turno, al menor crecimiento de la tasa de empleo de los trabajadores secundarios del $\mathrm{ABBC}$ de la de los $31 \mathrm{AU}$.

También resulta relativamente alta la tasa de desocupación de los trabajadores jóvenes del ABBC. En IV-2013 la tasa de desocupación de los jóvenes bahienses de los segmentos de edad 18-24 y 25-30 resultó un 50\% más alta que en los 31AU. Según los datos esto es explicado, en el caso, de los trabajadores de 18 a 24 años por una particularmente baja tasa de empleo mientras que para los del segmento de 25 a 30 años la explicación reside en una tasa de actividad relativamente elevada.

\section{OCUPACION Y DESOCUPACION A LO LARGO DE LA ULTIMA DECADA}

La tabla 3 ofrece un panorama general de la evolución de los volúmenes de ocupación y desocupación en el $\mathrm{ABBC}$ según las diferentes categorías para el conjunto de la década 2003/13. Se trata de proyecciones poblacionales de los datos muestrales relevados por la EPH. Dado el elevado error característico de estas estimaciones, nos limitamos a señalar sólo algunas tendencias que parecen imponerse con bastante claridad.

El número de ocupados se incrementó $16 \%$ en 10 años, lo que equivale a unos 18.500 puestos de trabajo. La tabla muestra que casi $80 \%$ de dicho incremento correspondió a la fuerza de trabajo masculina.

Además, entre ambos extremos del período, casi toda la creación neta de empleo se focalizó en jefes de hogar con un nivel educativo alto o medio. La composición por edades también se modificó ya que una parte sustancial del incremento neto de la ocupación correspondió a trabajadores mayores de 30 años. Por otra parte, el crecimiento de la ocupación llegó a su punto máximo en el IV- 
2010, es decir, hace más de cuatro años; desde entonces se mantiene estancado en torno de las 134.000 personas.

Respecto de la desocupación pueden señalarse los siguientes elementos. Su disminución en el período 2003 a 2013 fue de 8.000 personas, es decir 38\%. Por otra parte, el agotamiento del proceso de recuperación iniciado en 2003 resultó aún más prematuro que en lo referido a la ocupación. El siguiente gráfico muestra que el mínimo de desocupados se alcanzó en III-2008; desde entonces las personas sin trabajo fluctuaron en torno de 12.500 .

Tabla 3. Ocupados y desocupados. Aglomerado Bahía Blanca-Cerri. Cuarto trimestre de 2003 y de 2013. En miles*

\begin{tabular}{lcccccc}
\hline & \multicolumn{3}{c}{ Ocupados } & \multicolumn{3}{c}{ Desocupados } \\
\hline Aglomerado & 2003 & 2013 & Diferencia & 2003 & 2013 & Diferencia \\
Varones & 117,8 & 136,4 & 18,5 & 20,9 & 12,9 & -8 \\
Mujeres & 67,5 & 81,9 & 14,4 & 12,2 & 4,2 & $-8,1$ \\
Jefes de hogar & 50,3 & 54,5 & 4,1 & 8,7 & 8,8 & 0,1 \\
Cónyuges & 63,1 & 80,9 & 17,8 & 9,8 & 2,1 & $-7,7$ \\
Hijos & 27,9 & 29,5 & 1,6 & 3,6 & 6 & 2,4 \\
Edad 18-24 & 21,2 & 19 & $-2,2$ & 6 & 3,3 & $-2,6$ \\
Edad 25-30 & 20,2 & 13,8 & $-6,4$ & 5,4 & 4,7 & $-0,6$ \\
Edad 31-55 & 18,8 & 26,1 & 7,2 & 2,2 & 3,4 & 1,1 \\
Edad 56-65 & 65,2 & 74,8 & 9,5 & 8,6 & 3,9 & $-4,7$ \\
N. E. bajo & 10,4 & 19,1 & 8,8 & 3,5 & 0,6 & $-2,9$ \\
N. E. medio & 57,8 & 53 & $-4,8$ & 15 & 7 & $-8,1$ \\
N. E. superior & 43,5 & 50 & 6,5 & 5,3 & 3,6 & $-1,7$ \\
\hline
\end{tabular}

* Las sumas de las categorías de rol en el hogar y edad no resultan idénticas a los respectivos totales del aglomerado debido a la omisión de agrupaciones de reducido peso cuantitativo y sin interés para el análisis.

Fuente: EPH (INDEC) 
Gráfico 5. Número de ocupados

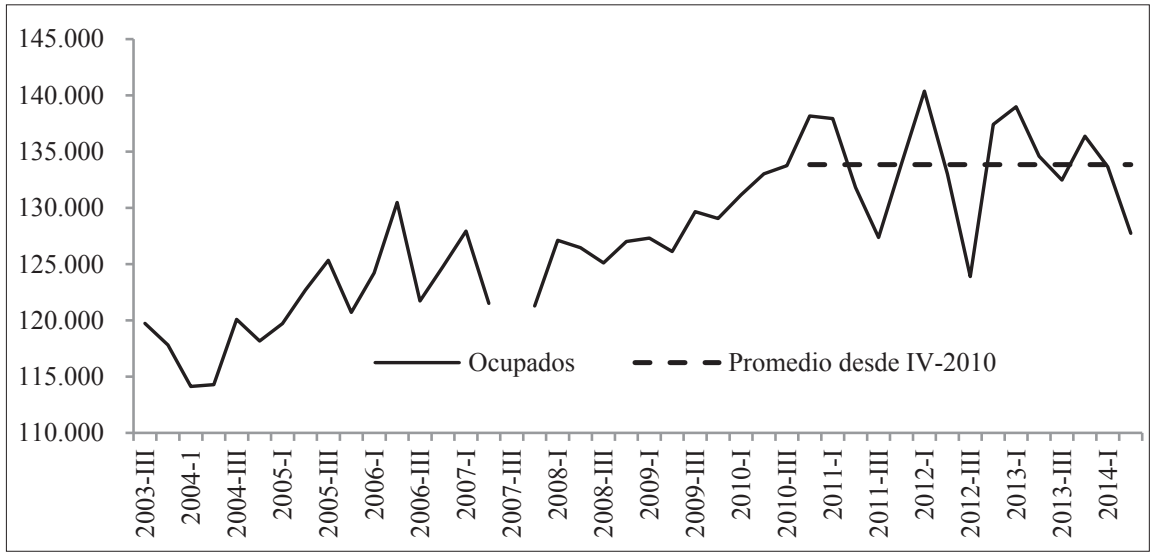

Fuente: EPH (INDEC)

Gráfico 6. Número de desocupados

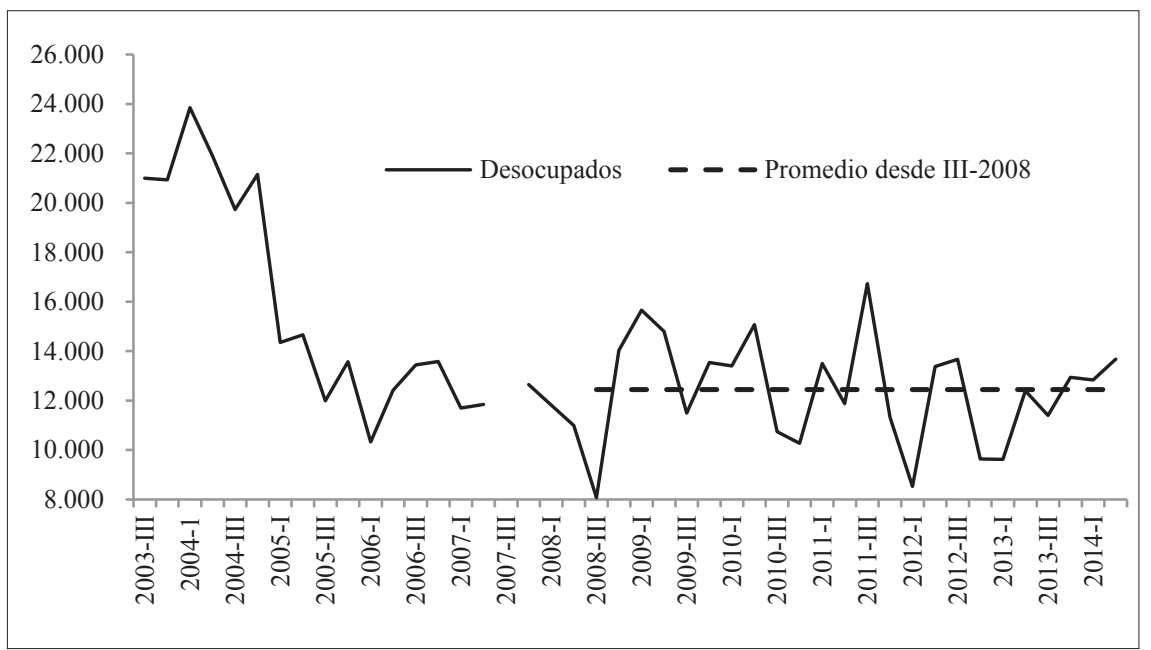

Fuente: EPH (INDEC)

En lo atinente a los diversos componentes de la fuerza de trabajo se pueden comentar algunos elementos de interés. La totalidad de la caída de la desocupación a lo largo de la década 2003/13 correspondió a los varones; el número de mujeres desocupados en 2013 resultó idéntico al de 2003. En 2013 había 67\% más cónyuges 
desocupados que en 2003; como ocurrió con el aumento de la ocupación, la caída de la desocupación se concentró en buena medida en jefes de hogar.

En 2013 había 1.100 jóvenes de 25-30 años desocupados más que en 2003 y el número de desocupados de jóvenes de 18-24 casi no se redujo en toda la década. El grueso de la disminución de la desocupación correspondió a trabajadores de 31-55 y en menor medida a los de 56-65. Por último, el número de desempleados con nivel educativo alto subió en casi 2.000 personas 5 . Curiosamente, se trata del mismo sector laboral que experimentó un gran aumento de los nuevos puestos de trabajo. Los datos muestran que los trabajadores de esta categoría tienen hoy mayor peso en la ocupación pero también en la desocupación, de la que actualmente representan casi un quinto.

El volumen de la fuerza de trabajo con nivel educativo bajo ha disminuido en casi $20 \%$ (entre ocupados y desocupados) a lo largo de la década y esta tendencia seguramente continuará. Con casi la mitad de los desocupados bahienses en 2013 con un nivel educativo medio y alto, los que no alcanzan dicho nivel seguirán sintiendo la presión de terminar los estudios secundarios para elevar sus chances de inserción laboral. Pese a esta tendencia, subsiste un muy voluminoso contingente de trabajadores con nivel educativo "bajo" con una fuerte gravitación en las fluctuaciones de la situación laboral. De hecho en 2003, 72\% de los desocupados del aglomerado tenía nivel educativo "bajo" y en 2007 aún representaba 54\%.

\section{PRECARIZACION LABORAL}

Diversos indicadores referidos a las características de la ocupación permiten entrever dos importantes rasgos de la situación laboral de la última década. El primero se refiere a la dramática profundidad de la crisis laboral generada por los cambios económicos e institucionales introducidos durante los años '90. Como muestra el siguiente cuadro, la precariedad de las relaciones laborales regía para casi la mitad de los ocupados del aglomerado.

El segundo rasgo es que la "reactivación" y "mejoría" de la situación laboral, lejos de restablecer la formalidad en el mercado de trabajo dejó un pesado y sustancial remanente de precariedad; al cabo de una década, se siguen registrando niveles inaceptables de precarización en todos los ítems. Este hecho es particularmente significativo si se considera que varios de los factores que en

5 Debido a la a gran variabilidad que muestra el número de trabajadores en las diversos niveles educativos este dato debe tomarse con cautela. 
la última década favorecieron el crecimiento económico (el colchón de ganancias empresarias generado por la devaluación de 2002, la evolución de los precios mundiales de las materias primas, etc.) han dejado de operar en los últimos años.

Tabla 4. Características de la ocupación principal.

Cuarto trimestre. En \%*

\begin{tabular}{lcc}
\hline & $\mathbf{2 0 0 3}$ & $\mathbf{2 0 1 3}$ \\
\hline Sin vacaciones pagas & 44 & 24 \\
Sin aguinaldo & 44 & 26 \\
Sin días pagos por enfermedad & 48 & 27 \\
Sin obra social & 46 & 27 \\
Sin descuento jubilatorio & 45 & 30 \\
Al cobrar no le dan recibo legal ${ }^{* *}$ & 35 & 28 \\
\hline Promedio simple $^{*}$ & 44 & 27 \\
\hline
\end{tabular}

* Los porcentajes se refieren al subconjunto de los ocupados a los que aplica la pregunta y no al total.

** Incluye a trabajadores que emiten factura.

Fuente: EPH (INDEC)

En el IV-2013 el 27\% indicado en la tabla equivalió a unas 33.000 que se comparan con unas 112.000 respuestas obtenidas para la correspondiente sección del formulario de encuesta de la EPH. Dada la brecha salarial que padecen las personas ocupadas en estas condiciones, es probable que todo o parte del contingente de ocupados precarios integre, junto a los desocupados, el amplio sector de la población del $\mathrm{ABBC}$ con ingresos por debajo de la línea de pobreza.

\section{CONCLUSIONES}

Conviene presentar las conclusiones bajo dos perspectivas diferentes. Por un lado, la evolución de los indicadores laborales del ABBC en la década 2003/13 y, por el otro, la comparación del mercado laboral local con el promedio de los $31 \mathrm{AU}$.

Respecto de la evolución del mercado de trabajo local a lo largo de la década la investigación ha logrado establecer los siguientes hechos salientes:

(a) La tasa de desocupación se redujo en 2003/7 gracias a la disminución de la oferta laboral y no al aumento de la tasa de empleo. Pero esta 
mejoría se agotó prematuramente de modo que la tasa de desocupación se estacionó en un nivel relativamente elevado en términos históricos. El número de desocupados dejó de disminuir en 2008.

(b) Dos sectores laborales muestran una desocupación más alta que la media del aglomerado; la fuerza de trabajo femenina y la de los jóvenes de hasta 30 años. La desocupación local también resulta elevada entre los trabajadores con nivel educativo bajo y en los últimos años se ha observado un deterioro de la situación de los trabajadores con estudios superiores.

(c) La disminución de la tasa de desocupación fue generalizada en 2003/7 porque en los sectores laborales en los que la tasa de empleo no se incrementó se observó una contracción de la tasa de actividad. Pero en el siguiente período (2007/13) sólo siguió bajando para la fuerza de trabajo "central" conformada por los varones adultos jefes de hogar; en los demás sectores la mayor tasa de empleo fue contrarrestada por un incremento de la tasa de actividad.

(d) En el período inmediatamente posterior al estallido de la crisis en 2002, la tasa de desocupación del ABBC tendió a disminuir para los diversos subsectores de la fuerza de trabajo. La tasa de empleo, en cambio, se incrementó para los sectores centrales de la fuerza de trabajo y decreció para los complementarios. Así, la recuperación de los años 2003/7 habilitó la recomposición de los sectores centrales de la fuerza de trabajo en detrimento de los llamados trabajadores "secundarios". Perdieron peso las mujeres, los jóvenes hasta 30 y los cónyuges.

Pero desde 2008 tuvo lugar una reversión de este proceso; la fuerza de trabajo "de reserva" retornó al mercado de trabajo. Esto coincidió con un estancamiento primero y deterioro después del poder adquisitivo de los salarios. Los datos muestran que, si bien se produjo un cierto aumento de la tasa de empleo en 2008/13 este sólo se tradujo en menor tasa de desocupación para los trabajadores centrales. Entre los secundarios, en cambio, o bien la tasa de empleo se mantuvo estable o bien su aumento resultó contrarrestado por el mencionado incremento de la tasa de actividad.

(e) La mayor parte de los puestos de trabajos creados en la década 2003/13 fueron ocupados por varones, jefes de hogar, mayores de 30 años, con nivel educativo intermedio o alto. La situación laboral ha dejado de mejorar hace varios años; la creación de puestos de trabajo alcanzó su pico máximo a fines de 2010 y la disminución de la desocupación se detuvo en el segundo semestre de 2008. 
La evolución del mercado de trabajo del ABBC en la década 2003/13 presenta varias características distintivas respecto del que se observó en el promedio de los aglomerados urbanos.

(a) A diferencia de lo ocurrido en el $\mathrm{ABBC}$, la caída de la tasa de desocupación en los 31AU en los años 2003/7 fue motorizada por el aumento de la tasa de empleo. En el período siguiente la evolución del $\mathrm{ABBC}$ volvió a ser más desfavorable; su tasa de desocupación dejó de disminuir mientras la del promedio urbano nacional continuó bajando, aunque a un menor ritmo que en el período previo.

(b) El ABBC tuvo en toda la década una mayor tasa de desocupación que los 31AU. En 2003/7 esto se debió a los trabajadores "centrales" mientras que en 2007/13 la brecha se debió a la elevada tasa de desocupación de los trabajadores "secundarios".

La investigación arroja dos conclusiones de orden general. Primero, no es posible afirmar que la recomposición de los años posteriores al estallido de 2002 haya revertido los fuertes desequilibrios laborales ocurridos durante la crisis de la convertibilidad en materia de desempleo y precarización. Segundo, al igual que a nivel nacional, el mercado de trabajo urbano bahiense ha dejado de mostrar mejoras significativas en los últimos años.

La superación de estos problemas laborales, tanto a nivel del $\mathrm{ABBC}$ como de los $31 \mathrm{AU}$ en general reclama transformaciones económicas, sociales y políticas de fondo.

\section{REFERENCIAS BIBLIOGRÁFICAS}

INDEC (2003). Encuesta Permanente de Hogares. Recuperado de http://200.51.91.245/argbin/RpWebEngine.exe/PortalAction?\&MODE $=$ MAIN\&BASE $=$ EPH_BASE_FINAL\&MAIN=WebServerMain.inl

INDEC (2007). Encuesta Permanente de Hogares. Recuperado de http://200.51.91.245/argbin/RpWebEngine.exe/PortalAction?\&MODE $=$ MAIN\&BASE $=$ EPH_BASE_FINAL\&MAIN=WebServerMain.inl

INDEC (2013). Encuesta Permanente de Hogares. Recuperado de http://200.51.91.245/argbin/RpWebEngine.exe/PortalAction?\&MODE $=$ MAIN\&BASE $=$ EPH_BASE_FINAL\&MAIN=WebServerMain.inl 
(C) 2015 por los autores; licencia otorgada a la Revista Estudios Económicos. Este artículo es de acceso abierto y distribuido bajo los términos y condiciones de una licencia Atribución-No Comercial 3.0 Unported (CC BY-NC 3.0) de Creative Commons. Para ver una copia de esta licencia, visite http://creativecommons.org/ licenses/by-nc/3.0/ 